\title{
La duración de la vida activa en la fuerza de trabajo del Gran Santiago 1960-2002
}

Rodrigo Rivero-Cantillano*

UNIVERSIDAD DE SANTIAGO

\section{RESUMEN}

Este artículo estudia la duración Vida Activa de la fuerza de trabajo en el mercado de trabajo del Gran Santiago entre 1960-2002. La duración de la Vida Activa corresponde a los ańos que en promedio permanecerá un trabajador en la actividad económica, bajo ciertos supuestos de participación y mortalidad. Con ese objetivo elaboramos de "tablas de vida activa”, cuya principal contribución es el cálculo de la esperanza de vida activa.

Palabras clave: Fuerza de trabajo, esperanza de vida activa, Gran Santiago

\begin{abstract}
:
This paper study the length of working life in the labor force of Greater Santiago between 1960-2002. The length of working life corresponds to the average years that a worker will remain in the economic activity, under certain assumptions of participation and mortality. With this objective we elaborate of "active life tables", whose main contribution is the calculation of the active life expectancy.
\end{abstract}

Keywords: Labor force, active life expectancy, Great Santiago

rodrigo.rivero@usach.cl* 


\section{INTRODUCCIÓN}

ste artículo estudia un aspecto específico de la de la fuerza de trabajo o población económicamente activa -aquella población que se encuentra disponible para la producción de bienes y servicios-, la duración de la vida activa. El concepto "vida activa" no es nuevo, al menos desde el siglo XVIII, actuarios, economistas y sociólogos, han intentado llegar a alguna medida del valor "capitalizado" de la vida humana, han hecho estimaciones explícitas o implícitas de la duración de la "vida productiva". Este concepto ilustra no sólo la relevancia de los factores demográficos, sino que adapta la técnica de la tabla de vida a una medida de la vida laboral. Por lo tanto, la duración de la vida activa es entendida como los años que en promedio permanecerá un trabajador en la actividad económica, bajo ciertos supuestos de participación y mortalidad.

El estudio de la fuerza de trabajo nos proporciona una excelente ilustración de la importancia subyacente de los factores demográficos para muchas otras ciencias sociales. Por ejemplo, las características demográficas de una población son factores íntimamente ligados a su participación económica. En particular el sexo, la edad, la residencia urbana o rural, el estado civil, o incluso, el número de hijos en el caso de las mujeres y el ingreso familiar en el caso del trabajo infantil, entre muchos otros, influyen sobre la oferta de trabajo (Elizaga, 1979; Pardo, 1988; Larrañaga, 2006).

En Chile, durante la segunda mitad del siglo XX, la fuerza de trabajo experimentó cambios en su volumen, características educativas, esperanza de vida, composición por edad y sexo, y desde una perspectiva económica, en su estructura ocupacional. En síntesis, en la historia económica de la población chilena, destacan cambios importantes en relación al tamaño y naturaleza de la fuerza de trabajo que han tenido efectos presumiblemente sobre la duración de su vida activa (Rivero-Cantillano, 2016).

A pesar de la importancia de este factor, existe muy poca evidencia acerca de las tendencias históricas en la duración de la vida activa en Chile. En las últimas décadas, gracias al rápido avance del envejecimiento, se ha prestado mayor atención a los problemas económicos de la dependencia en la vejez, las estimaciones de la duración de la vida activa, en comparación con la duración total 
de la vida, son pertinentes a este problema. En este sentido, es importante conocer las consecuencias de efecto combinado del cambio demográfico y los cambios en los niveles de participación sobre la duración de la "vida productiva" en perspectiva histórica. Además, es interesante desde varios puntos de vista, puesto que en teoría puede pensarse que la prolongación de la vida activa representa una mayor contribución económica al conjunto de la sociedad, o también, aumentar los beneficios para la seguridad social, esto último adquiere mayor relevancia con una mirada hacia el futuro.

Para medir la vida activa, recurrimos a la elaboración de "Tablas de Vida Activa" -en adelante TVA-, que nos permiten conocer la duración de la vida activa así como los flujos de ingresos y retiros de la actividad económica. Las TVA, dependen de dos factores principales, primero, el efecto de la mortalidad, y en segundo lugar, los niveles de participación económica. Respecto a este último, al estar elaboradas con los datos de población activa e inactiva observadas en cada momento, integran los cambios de coyuntura macroeconómica que tienen gran relevancia en la participación de la fuerza de trabajo. El método de TVA fue desarrollado por Wolfbein (1949), corresponde al método tradicional basado en la prevalencia observada de las tasas de participación económica. Algunas contribuciones se han hecho para mejorar este método desde su concepción original por Wolfbein (Durand y Miller, 1969; Camisa, 1969; Somoza, 1970; Rincón, 1977); no obstante, el supuesto fundamental ha prevalecido. Con ese objetivo nuestras fuentes primarias serán, en primer lugar, la Encuesta de Ocupación y Desocupación del Gran Santiago, especializada en el registro de la población económicamente activa -en adelante PEA-, de sus tendencias de participación, ocupación, desocupación, así como sus características, realizada de forma ininterrumpida desde 1957 hasta hoy. En segundo lugar, las Tablas de Mortalidad (INE, 2004), desde donde obtendremos los patrones históricos de mortalidad a la cual se ha visto expuesta la población del Gran Santiago.

El resto del trabajo continúa de la siguiente manera. En la segunda parte se expone la metodología y fuentes de datos, la tercera parte, describe los patrones históricos de participación de la fuerza de trabajo del Gran Santiago, en la cuarta parte, se presentan los 
resultados de las TVA: la duración de la vida activa de la fuerza de trabajo del Gran Santiago entre 1957 y 2002, en la quinta parte se exponen las consideraciones finales. Por último, se cierra el trabajo con una entrega detallada de las funciones de la TVA y un apéndice metodológico para la elaboración de TVA.

\section{Metodología: la Tabla de Vida Activa}

La esperanza de vida activa y de vida inactiva, cuando se analizan a través del tiempo, reflejan el influjo combinado de los cambios en los indicadores demográficos, sociales y económicos que influyen en las tasas de participación. Gracias a la simplicidad de sus supuestos, permite aproximarnos al estudio de los componentes de los cambios en la fuerza de trabajo, y en función de los mismos, estimar los montos de inversiones requeridas para integrar productivamente a los trabajadores entrantes en los mercados de trabajo, así como hacer las previsiones conducentes a los jubilados y pensionados (Morelos, 1996).

Los métodos para construir las TVA se pueden clasificar en tres tipos, la tabla de vida activa basada en la prevalencia observada, la tabla de vida activa de múltiples decrementos y la tabla de vida activa de incrementos y decrementos. Entre todos, el más utilizado, es el método de las tablas de vida laboral basadas en la prevalencia observada del fenómeno, que fue propuesto por Durand (1948), modificado por Sullivan (1971), con tanto éxito que pasó a denominarse método Sullivan. Este método ha sido y sigue siendo muy utilizado para realizar estudios comparativos de estados de actividad entre países o momentos históricos dentro de un mismo país además de otros usos (Gutiérrez de Mesa, 2010).

La construcción de una tabla de vida activa, dadas las limitaciones de la información disponible, implica asumir una serie de supuestos fundamentales, entre los cuales están (Partida-Bush, 1996):

a) La participación de la población en la actividad económica se da dentro de un intervalo de edad previamente definido como la edad de ingreso - que puede ser la edad legal o una cercana a ella- y retiro $(a, d)$. Fuera de ese intervalo la actividad es nula. Estas edades de ingreso y retiro pueden variar de país en país o incluso dentro de un mismo país en dos momentos históricos. 
b) El ingreso a la actividad económica se da solo cuando sus edades están comprendidas en un intervalo $(a, b)$, que es de menor duración que la vida activa; donde b corresponde, a la edad en la que la unción de participación alcanza su valor máximo. Por otro lado, dentro de este intervalo solo se producen retiros por efecto de la mortalidad.

c) Los retiros de la actividad económica se producen en personas comprendidas en el intervalo de edades (b, d). En este intervalo no hay ingresos a la actividad económica. El punto d, se define arbitrariamente, pero en general varía de acuerdo al nivel de desarrollo y del tipo de población que se estudie. Generalmente tiende a ser menor cuanto más alto sea el nivel de desarrollo del país analizado. $^{2}$

d) Por último, debido a la falta de información adecuada, se considera que la mortalidad de la población activa e inactiva son iguales. Supuesto que puede ser entendido más bien como una restricción.

La interpretación de la tabla de vida activa es simple, representa el número medio de ańos que permanecería en la condición de activo o inactivo un individuo de una generación ficticia que a lo largo de su vida experimentaran las mismas condiciones de actividad y mortalidad que el conjunto de la población real en el periodo de referencia (Gutiérrez de Mesa, 2010).

En otras palabras, la tabla de vida activa refleja la dinámica de participación de una cohorte hipotética, sometida durante toda su vida a las condiciones de mortalidad y participación implícita en las funciones utilizadas.

La aplicación de la ecuación compensadora a la cohorte de la tabla de vida activa sería:

$$
l_{x+n}^{a}=l_{x}^{a}+{ }_{n} H_{x}^{i a}-{ }_{n} H_{x}^{a i}-{ }_{n} d_{x}^{a} y l_{x+n}^{i}=l_{x}^{i}+{ }_{n} H_{x}^{a i}-{ }_{n} H_{x}^{i a}-{ }_{n} d_{x}^{i}
$$

Donde $l_{x}^{a}$ corresponde a los sobrevivientes activos y $l_{x}^{i}$ a los sobrevivientes inactivos. Siendo: ${ }_{n} H_{x}^{i a}$ los ingresos a la actividad, ${ }_{n} H_{x}^{a i}$ los retiros, ${ }_{n} d_{x}^{a}$ las defunciones de activos $\mathrm{y}{ }_{n} d_{x}^{i}$ las de inactivos (Partida-Bush, 1996), (ver apéndice metodológico). 


\section{FuENTES Y BASES DE DATOS}

Un insumo fundamental para la elaboración de las TVA es contar con "tablas de mortalidad". En este trabajo dispondremos de las tablas de mortalidad publicadas por el Instituto Nacional de Estadísticas (INE, 2004), para los periodos 1960-61, 1969-70, 1980-85, 1991-92 y 2001-02, a nivel nacional y regional. ${ }^{3}$ Desde aquí obtendremos las siguientes funciones, probabilidades de muerte $\left(\mathrm{n}_{\mathrm{x}}\right)$, sobrevivientes de la cohorte ficticia $\left(l_{x}\right)$, años personas vividos $\left(L_{n}\right)$, sobrevivencia $\left({ }_{n} p_{x}\right)$, defunciones $\left(d_{n}\right)$ y la esperanza de vida $\left(e_{x}\right)$. Cabe mencionar que el registro de una tabla de mortalidad o una tabla de vida, aunque tiene un origen demográfico, su conocimiento interesa a un amplio conglomerado de investigaciones en ciencias sociales, puesto que corresponde a un documento histórico que refleja indirectamente el nivel de vida de una época determinada, siendo el soporte de una gran variedad de temas relacionados con la población, desde el sector de la salud hasta la economía (INE, 2004).

Por otro lado, a diferencia de la mortalidad, donde se cuenta con registros, para la PEA solo se disponen de los censos de población, encuestas de hogares por muestreo y en los desgloses de los censos económicos en establecimientos industriales, comerciales y de servicios, estos últimos con grandes limitaciones. De esta manera, recurriremos a la Encuesta de Ocupación y Desocupación en el Gran Santiago -en adelante EOD-, publicada por el Centro de Microdatos de la Universidad de Chile de forma ininterrumpida desde 1957 hasta hoy, característica que le convierte en un patrimonio estadístico para el país. La población objetivo de la EOD incluye a todas las personas mayores de 14 años que residen en viviendas particulares habitadas (o habitables) ubicadas en las 32 comunas urbanas de la provincia de Santiago, además de las comunas de Puente Alto y San Bernardo. La cobertura de la encuesta corresponde a las 34 comunas del Gran Santiago, lo que corresponde a un $87,6 \%$ de la población total de la Región Metropolitana (Centro de Microdatos, 2016). En efecto, el diagnóstico y la evidencia empírica proporcionada por la EOD durante su larga trayectoria, han posibilitado la identificación de momentos de quiebres en el mercado laboral chileno constituyendo, históricamente, una de las principales herramientas para el análisis del mercado laboral. Desde esta valiosísima fuente de datos 
obtendremos los promedios de población activa e inactiva por edad y sexo para los periodos siguientes 1960-61, 1969-70, 1980-85, 1991-92 y 2001-02, mismos periodos para los que contamos con tablas de mortalidad. ${ }^{4}$

\section{DinÁMiCAS DE PARTICIPACión EN LA FUERZa de TRABAJO DEL GRAN} SANTIAGO I 957-2006

En perspectiva histórica, los patrones de participación económica de la población chilena, se han visto influenciados por las grandes transformaciones tanto en la estructura económica como en la estructura demográfica, que desde mediados del siglo $\mathrm{XX}$, han dado origen a significativos cambios en la naturaleza, estructura y localización de la fuerza de trabajo. A medida que la economía chilena se diversificaba, las ciudades se transformaron en un gran atractivo para los trabajadores en búsqueda de mejores expectativas de vida, de manera que estas concentraron una creciente oferta de trabajo. ${ }^{5}$ La población en edad de trabajar -entre los 15 y 64 años de edad- residente en centros urbanos pasó del $63,4 \%$ en el censo del 1952, al 70,7 y 77,2\% en los censos de 1960 y 1970 respectivamente. Este proceso continuó hasta principio de los ochenta, aunque con menor intensidad, en el censo de 1982 el 83,3\% de la población en edad de trabajar residía en entornos urbanos, cifra que se mantuvo prácticamente sin cambios hasta el censo de 1992 con un 82,4\%, dando por finalizado el proceso de relocalización de la población en edad de trabajar. ${ }^{6}$

Sin duda que la combinación de factores demográficos, sociales y económicos jugaron un importante papel en los cambios observados en la participación. Como es bien sabido, cada forma de organización económica y social lleva implícita una forma de división social del trabajo, que hace que la PEA adquiera una composición diferente según sea su edad, sexo, calificación, ocupación o área en el que se ocupa (Rincón, 1977; Paredes y González 2002). En este sentido, el desplazamiento de la población transcurre desde un entorno rural de baja productividad y alta participación, a otro de carácter urbano cuya productividad es mayor, no obstante, su participación se ve condicionada por aspectos como la edad, el nivel educativo o el género. 
Por otro lado, la edad es una de las variables más importantes para el análisis de la participación en la actividad económica, en ella, se reflejan las transformaciones socioeconómicas de una población. Por ejemplo, entre los más importantes encontramos aspectos tales como las tendencias de fecundidad y mortalidad, leyes sobre la edad mínima de ingreso a la actividad económica y sobre la edad de retiro, las mejoras en los sistemas de educación, que son factores que modifican la edad de vinculación de la población al sistema de producción (Rincón, 1977; Hayward y Grady, 1990). Como podemos observar en el cuadro 1, los cambios más importantes se aprecian en los grupos de edades de inicio y de cierre de la vida activa, entre los 15-19 y 20-24 ańos por un lado, y entre los 55-59 y 60-64 años. En perspectiva comparada urbano-rural, observamos que en el medio urbano, los jóvenes retrasan su ingreso al mercado de trabajo, contraste que se hace más evidente en el grupo 15-19 años

Cuadro 1. Chile: Tasas de participación económica por grupo de edad

\begin{tabular}{|c|c|c|c|c|c|c|c|c|c|c|c|c|}
\hline & \multicolumn{3}{|c|}{1952} & \multicolumn{3}{|c|}{1960} & \multicolumn{3}{|c|}{1970} & \multicolumn{3}{|c|}{1982} \\
\hline & Total & Urbano & Rural & Total & Urbano & Rural & Total & Urbano & Rural & Total & Urbano & Rural \\
\hline 15 a 19 & 47,0 & 43,4 & 52,3 & 42,2 & 38,8 & 49,6 & 30,7 & 27,3 & 41,6 & 25,0 & 21,4 & 41,9 \\
\hline 20 a 24 & 62,2 & 63,5 & 59,7 & 60,9 & 61,4 & 59,6 & 57,4 & 56,8 & 59,5 & 55,9 & 55,2 & 59,3 \\
\hline 25 a 29 & 61,9 & 63,7 & 58,4 & 60,9 & 62,1 & 58,0 & 61,6 & 62,5 & 58,3 & 63,4 & 64,2 & 59,2 \\
\hline 30 a 34 & 61,8 & 63,2 & 59,0 & 59,7 & 60,5 & 57,3 & 60,3 & 61,2 & 57,1 & 63,4 & 64,4 & 58,3 \\
\hline 35 a 39 & 60,8 & 62,1 & 58,3 & 58,4 & 59,3 & 56,0 & 59,6 & 60,4 & 56,4 & 62,4 & 63,4 & 57,0 \\
\hline 40 a 44 & 62,7 & 63,6 & 61,2 & 58,2 & 58,6 & 57,3 & 58,8 & 59,3 & 56,9 & 60,6 & 61,4 & 56,4 \\
\hline 45 a 49 & 60,0 & 60,1 & 60,0 & 56,2 & 55,7 & 57,4 & 56,1 & 56,3 & 55,7 & 56,7 & 57,1 & 54,8 \\
\hline 50 a 54 & 58,1 & 56,5 & 60,7 & 53,3 & 51,3 & 58,0 & 52,1 & 51,0 & 55,3 & 51,0 & 50,5 & 53,7 \\
\hline 55 a 59 & 55,7 & 52,8 & 60,5 & 49,3 & 46,1 & 56,7 & 47,0 & 44,2 & 54,8 & 43,0 & 41,2 & 51,0 \\
\hline 60 a 64 & 50,6 & 46,0 & 57,5 & 43,5 & 38,7 & 53,7 & 39,6 & 35,4 & 51,7 & 33,8 & 30,8 & 46,2 \\
\hline
\end{tabular}

Fuente: Censos de Población de Chile, INE (1952-1982).

Entre los factores que se encuentran detrás del retraso al ingreso al mercado de trabajo está la mayor escolarización y formación profesional de la población urbana, hecho que actúa reduciendo las tasas de actividad de los grupos 15-19 y 20-24, no obstante, en el largo plazo, los efectos de la mayor educación y calificación significará un aumento en la tasa de participación económica a partir de los 25 ańos de edad, proceso de especial intensidad entre las mujeres. ${ }^{7}$ Junto con el progreso del sistema educativo -en cuanto a su 
acceso y duración de la obligatoriedad- la población en edad escolar vio aumentar sus oportunidades reales de educarse, reduciendo de forma considerable el riesgo de pasar a formar parte de la fuerza de trabajo a temprana edad, que por cierto, es uno de los prerrequisitos fundamentales para el desarrollo. Respecto de las personas mayores, en el medio rural, el retiro se ve disminuido frente a la necesidad de la subsistencia, con lo cual gran parte de la población pese a cumplir la edad legal de retiro permanece en activo, realidad que se observa en menor medida la población urbana gracias a la cobertura del sistema de seguridad social. ${ }^{8}$

Respecto de la situación en el Gran Santiago, podemos comprobar la intensidad del declive en las tasas de participación económica en los grupos de 15-19 y 20-24 años, siendo ésta mucho más marcada en el grupo de 15-19 años, si en el periodo 195761 la tasa de actividad económica del grupo 15-19 ańos era de un $37 \%$, al inicio del siglo XXI alcanzaba un $17 \%$ en $2002-2006$, una reducción de veinte puntos porcentuales. Por otro lado, observamos un aumento generalizado en las tasas de participación económica, que cobra mayor intensidad a partir de la década de 1980, siendo especialmente significativa en los grupos de edades más avanzadas (ver cuadro 2). edad

Cuadro 2. Gran Santiago: Tasas de participación económica por

\begin{tabular}{ccccccccccc}
\hline & $1957-61$ & $1962-66$ & $1967-71$ & $1972-76$ & $1977-81$ & $1982-86$ & $1987-91$ & $1992-96$ & $1997-01$ & $2002-06$ \\
\cline { 2 - 9 } $15-19$ & 37,0 & 35,2 & 30,3 & 23,0 & 20,5 & 18,2 & 17,9 & 18,2 & 17,9 & 17,4 \\
$20-24$ & 64,5 & 63,1 & 64,1 & 60,6 & 59,6 & 59,6 & 59,4 & 59,4 & 58,4 & 58,0 \\
$25-29$ & 66,4 & 66,3 & 68,8 & 68,3 & 69,4 & 70,0 & 71,4 & 73,0 & 76,7 & 76,6 \\
$30-34$ & 66,3 & 64,3 & 67,0 & 69,0 & 70,0 & 70,7 & 73,2 & 74,9 & 78,5 & 79,3 \\
$35-39$ & 64,3 & 63,7 & 66,4 & 66,9 & 68,0 & 70,7 & 71,7 & 74,5 & 77,6 & 78,4 \\
$40-44$ & 64,7 & 64,6 & 65,2 & 65,4 & 69,9 & 69,2 & 73,5 & 73,0 & 78,3 & 78,3 \\
$45-49$ & 61,8 & 60,6 & 61,6 & 64,7 & 63,0 & 63,2 & 68,1 & 72,4 & 77,7 & 77,2 \\
$50-54$ & 55,4 & 54,8 & 57,9 & 55,4 & 56,8 & 56,3 & 62,4 & 66,1 & 73,3 & 73,6 \\
$55-59$ & 50,1 & 47,8 & 51,7 & 46,5 & 44,3 & 44,7 & 50,5 & 57,2 & 65,0 & 65,9 \\
$60-64$ & 41,9 & 36,2 & 40,2 & 36,3 & 36,7 & 31,9 & 33,2 & 40,2 & 50,3 & 53,6 \\
\hline
\end{tabular}

Fuente: Elaboración propia a base de Encuesta de Ocupación y desocupación del Gran Santiago, Universidad de Chile.

Tanto la reducción de las tasas de participación económica en 
los grupos jóvenes como el aumento de las mismas en los grupos de edades avanzadas, son hechos que, entre otros factores, están relacionados con las mejoras en la esperanza de vida a lo largo del periodo estudiado. En el caso de los jóvenes, más años de esperanza de vida actúan como incentivo a una mayor capacitación, por otro lado, en el caso de los trabajadores mayores más años de vida motivan a mantenerse como activos ya sea por incentivos o necesidad económica. ${ }^{9}$ De esta manera, observamos cambios importantes en los patrones de participación económica, que sin duda han tenido efectos sobre la extensión del "periodo productivo" a lo largo del curso de vida de los trabajadores.

\section{TRANSICIONES ENTRE ESTADOS DE LA VIDA ACTIVA E INACTIVA}

La PEA como cualquier otra sub-población abierta, experimenta un proceso continuo de crecimiento y renovación. Durante un periodo de tiempo dado, nuevas personas entran a formar parte de ella, mientras que al mismo tiempo otro grupo sale de la misma por muerte, por el retiro de la vida activa o por otros motivos, como por ejemplo un retiro voluntario o por motivos de discapacidad (Elizaga 1979). La edad juega un rol fundamental en los patrones de ingresos y retiros, pues las transiciones entre los estados de actividad e inactividad se dan con una gran intensidad en edades extremas, desde la inactividad hacia la actividad entre los jóvenes, y desde la actividad hacia la inactividad en los adultos mayores.

Las diferencias observadas en las probabilidades de transición de un estado a otro para los distintos periodos, dan cuenta del comportamiento de las tasas de actividades e inactividad observadas, así como la incidencia de la mortalidad entre los activos. En este sentido, comprobamos que, en el mercado de trabajo del Gran Santiago, la probabilidad de transición desde la inactividad hacia la actividad ( $\left.\mathrm{p}^{\mathrm{i} a}\right)$ experimenta en ambos sexos un crecimiento constante desde el periodo 1960-61 a 2001-02, transversal a todas las edades (ver anexo metodológico). No obstante, se advierten diferencias entre los sexos pues la probabilidad de incorporarse a la vida activa es mayor en los hombres que en las mujeres, como es evidente dada la mayor participación de los hombres en el mercado de trabajo. Por otro lado, detrás de las diferencias observadas están las distintas 
características socioeconómicas, culturales y de la estructura económica que influyen en la desigual incorporación de hombres y mujeres al mercado de trabajo, (ver cuadro A.1. en Apéndice).

Respecto de las probabilidades de mantenerse como activos $\left(\mathrm{p}^{\mathrm{a} a}\right)$, como es de esperar, en general disminuyen con el avance de la edad tanto hombres como en mujeres, no obstante, los comportamientos por sexo difieren considerablemente, (ver Gráfico 1 y Cuadro A.1. en apéndice).

Gráfico 1. Gran Santiago: Transición entre estados: probabilidad de mantenerse en actividad por edad y sexo
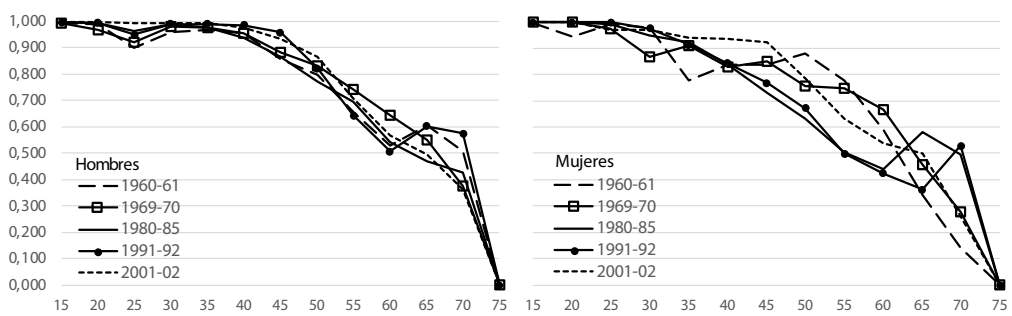

Fuente: Elaboración propia a base de Encuesta de Ocupación y desocupación del Gran Santiago, Universidad de Chile. Tablas de Mortalidad INE (2004).

Como podemos observar, los mayores cambios en la permanencia en la actividad las notamos entre las mujeres, donde se aprecia un aumento en las probabilidades de transición a través de los periodos. Estos cambios son el reflejo del crecimiento de la participación de las mujeres en el mercado de trabajo. El cambio más significativo lo observamos entre las mujeres de treinta a cuarenta años en los periodos 1960-61 y 1969-70. Respecto de la tendencia general, a partir de los 14 años edad la EOD registra tasas de participación, las que aumentan a medida que avanza la edad, alcanzando la tasa más alta de participación entre los grupos de edad de 30 y 35 ańos. A partir de esta edad, se abre un proceso gradual de retiro, ya sea voluntario o por razones de salud, y que culmina en el grupo de edad de 80 y más años. ${ }^{10}$ 
LOS FLUJOS DE INGRESOS A LA ACTIVIDAD Y RETIROS DEL MERCADO de trabajo del Gran Santiago

La dinámica de la PEA está constituida por los movimientos de ingreso de trabajadores desde una edad inicial de incorporación -generalmente la mínima legal ${ }^{11}$ - hasta la edad de participación máxima -generalmente definida por la edad legal de jubilación-.

Eventualmente entre estas dos edades, se producen retiros anticipados por causa de muerte, invalidez -que puede ser momentáneo o no- o retiro voluntario, no obstante, la mejora de los niveles de mortalidad hace que los retiros por defunciones disminuyan a través del tiempo y la extensión de la esperanza de vida a edades avanzadas hace que una parte de este grupo de población permanezca voluntariamente como activas más allá de la edad legal de jubilación. Por otro lado, además de este proceso general de entradas y retiros, que ocurren principalmente en edades marginales, algunas personas reingresan después de un tiempo de inactividad. En efecto algunas personas salen de la actividad y reingresan después de un tiempo, otras salen por invalidez y en el caso de las mujeres existen retiros por causa de factores como la nupcialidad y maternidad (Elizaga, 1979; Partida-Bush, 2014).

En este sentido, como hemos visto a través de las probabilidades de transición, la gran mayoría de los que ingresan al estado activo son jóvenes, y a su vez, la mayor parte de los que salen de la actividad son de edad avanzada. Por otro lado, el aumento de la esperanza de vida ha llevado a un número no menos importante de trabajadores a permanecer en el mercado de trabajo aun habiendo superado la edad legal para el retiro. De esta manera, las tasas de retiro crecen hasta los 65 ańos y luego disminuyen influidas por la permanencia de trabajadores que pese a que cumplen la edad requerida para el retiro permanecen como activos, probablemente no gozan de una prestación económica por jubilación que les permita abandonar la actividad, por lo que deben mantenerse en la actividad algunos años más hasta que las condiciones de salud lo permitan. ${ }^{12}$ El rápido ascenso en las tasas de retiro en el último grupo de edades (75-79) se debe a que impusimos la condición de participación nula a partir de los ochenta y más años.

Las tasas de ingreso a la actividad en el mercado de trabajo del 
Gran Santiago, nos revelan una extensión del periodo de ingreso entre 1960 y 2002 visto a través de las edades. La edad media de ingreso a la actividad ha aumentado de acuerdo con la prolongación del periodo de escolaridad y formación profesional. No obstante, esta postergación, luego se ve compensada con una mayor tasa de ingreso a partir de los 20 ańos, tanto en hombre como en mujeres, razón por la cual a través de los distintos periodos crecen las incorporaciones hasta el grupo de 30-34 años, dinámica que no observamos en el periodo 1960-61. Por otro lado, cuando observamos las tendencias de las tasas de retiro observamos que en promedio estas son mayores en las mujeres, aunque, en el largo plazo, se evidencia una convergencia en lo patrones observados en las tasas de retiro de ambos sexos (ver gráfico 2 y cuadro A2 en apéndice).

Llama la atención la fuerte tendencia de abandono creciente de las mujeres entre 30 y 39 ańos en el periodo 1960-61, el que puede estar asociado a las incompatibilidades entre el trabajo productivo y reproductivo en una época caracterizada por los altos niveles de fecundidad, al mismo tiempo se observa una reincorporación al mercado de trabajo entre los 40 y 54 años, a partir de aquí las edades típicas de retiro hacen crecer las tasas. En general, la característica principal del retiro tanto en hombres como en mujeres es la influencia creciente que ejerce la edad a medida que se acerca a los 65 ańos.

Como podemos observar, en el mercado de trabajo del Gran Santiago, el flujo de ingresos y retiros muestran un patrón de comportamiento que difiere significativamente antes y después de la década de los ochenta (ver gráfico 2 y cuadro A2 en apéndice). Hasta los 65 ańos de edad, posiblemente el efecto de la mortalidad en descenso haya podido contrarrestar, en parte, la influencia de la tasa de participación declinante de la mano de obra entre mediados del siglo XX y la década de 1980. 
Gráfico 2. Gran Santiago: Tasas de ingreso y retiro de la actividad, 1960-2002

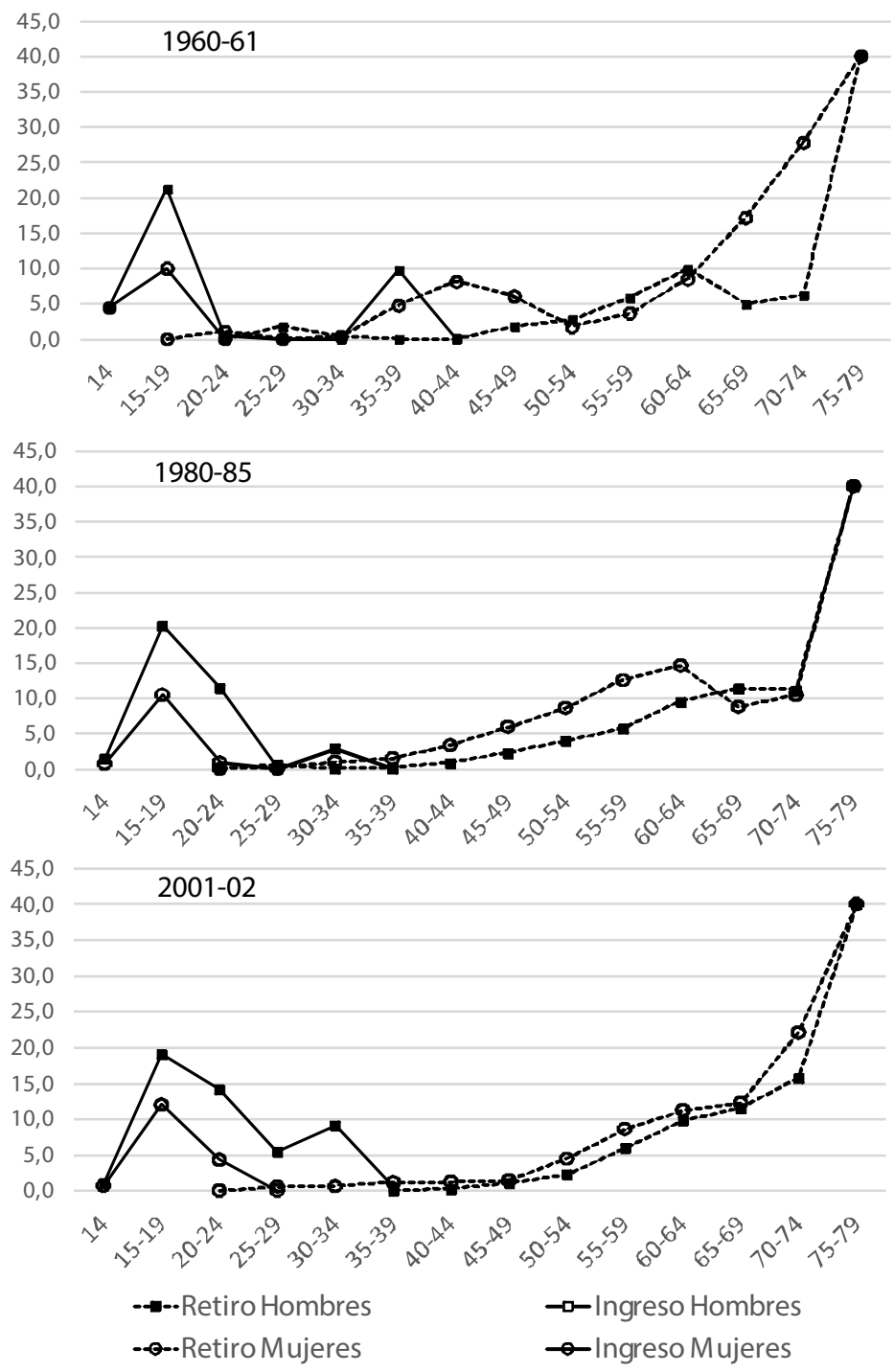

Fuente: Elaboración propia a base de Encuesta de Ocupación y desocupación del Gran Santiago, Universidad de Chile. Tablas de Mortalidad INE (2004). 
El progreso social y económico que hemos observado a través de las tasas de mortalidad y la esperanza de vida, han hecho que el papel jugado por la mortalidad en la dinámica de la fuerza de trabajo haya sido la reducción del número relativo de trabajadores nuevos requeridos para llenar las vacantes producidas por las defunciones, proceso que se consolida a medida que transcurre la segunda mitad del siglo XX. En los periodos de 1960-61 y 1969-70, los retiros son ampliamente superiores a los ingresos, dejando holgura para la ocupación efectiva de la población activa entrante. No obstante, a pesar del excedente de retiros en el periodo 1969-70. Tras estos dos periodos, a partir del periodo 1980-85, ingresos y retiros tienden a igualarse, siendo esta tendencia aún mayor entre las mujeres. Esta situación supone una mayor capacidad de adaptabilidad de la PEA, al mismo tiempo significa que deberían hacerse mayores esfuerzos por crear más puestos de trabajos, ya que el número de las vacantes por retiro es cada vez menor (cuadro A2 en apéndice). Presión que por el lado demográfico se suma a la coyuntura de grave crisis económica durante el periodo 1980-85 y a la lenta recuperación de puestos de puestos de trabajo una vez superada la coyuntura de crisis en los noventa (Meller, 1984; Cowan, et, al. 2005).

\section{Duración de LA VIDA aCTIVA DE LA FUERZa DE TRABAJO EN EL Gran Santiago i 960-2002}

Las tasas de participación son un indicador adecuado para conocer la dinámica de la fuerza de trabajo, no obstante, están influenciadas por la estructura de edad y por los efectos del cambio en el ciclo económico. De esta manera, para comprender mejor los las potencialidades del mercado de trabajo es necesario conocer el número medio de ańos que permanece en la actividad económica la fuerza de trabajo, bajo el supuesto que los niveles de participación en determinando momento permanecieran sin cambios a lo largo de la vida del trabajador. Al comparar los cambios en perspectiva histórica podemos comprender las condiciones del contexto demográfico y económico del momento analizado.

La duración de la vida activa la podemos determinar a través de los años brutos y netos de vida activa, que sintetizan -bajo los supuesto ya señalados- el total de años que permanecería como 
activo un individuo de una cohorte ficticia que presentase a lo largo de su vida las mismas tasas de actividad que la población real en un momento determinado. Los años brutos representan el número medio de años de económicamente activos de trabajadores y trabajadoras que no mueren antes de la edad de retiro, mientras que los ańos netos, incluyen la interrupción de la vida activa por la muerte antes de alcanzar la edad de retiro (ver apéndice metodológico). De esta manera, los años brutos de vida activa dependen únicamente de la edad a la que se comienza a trabajar y de la edad de retiro, mientras que los años netos son también afectados por la mortalidad (Elizaga, 1970; PartidaBush, 1996; 2014).

Considerando los factores que influyen en la duración de la vida activa, es de esperar que a mayor grado de desarrollo social y económico al que se expone una población, menor será el número de años brutos de vida activa, mientras que en los ańos netos de vida activa, la tendencia esperada es la opuesta, aumentado conforme aumenta el nivel de desarrollo. ${ }^{13}$ Respecto a esto último, con el avance del desarrollo social y económico de los países se observa por un lado, la reducción de la proporción de los años de vida activa de los trabajadores en relación a la duración total de su vida, y por otro lado, a pese a lo anterior, la extensión de la duración de la vida activa neta en el mercado laboral, gracias la mejora en los indicadores biosociales de bienestar ${ }^{14}$, como la reducción de la mortalidad, el aumento de la esperanza de vida, y consecuentemente, el incipiente envejecimiento de la población.

Visto en perspectiva histórica los ańos netos de vida activa tenderán a alcanzar o igualarse a un límite superior, que son los años brutos de vida activa, tendencia que podemos observar en el mercado de trabajo del Gran Santiago entre los periodos 1960-61 a 2001-02. Por otro lado, la diferencia entre años brutos y netos de vida activa equivales a los años de trabajo perdidos a causa de la mortalidad, los cuales se reducen de forma constante (ver Cuadro 3 y Cuadro A3 en apéndice). ${ }^{15}$ 
Cuadro 3. Gran Santiago: Años brutos y netos de vida activa a la edad de 15 años

\begin{tabular}{ccccccc}
\hline & \multicolumn{3}{c}{ Años brutos de vida activa } & \multicolumn{2}{c}{ Años netos de vida activa } & \multicolumn{2}{c}{ Esperanza de vida e $\mathbf{e}_{\mathbf{1 5}}$} \\
& Hombres & Mujeres & Hombres & Mujeres & Hombres & Mujeres \\
\cline { 2 - 7 } $1960-61$ & 42,1 & 21,1 & 34,4 & 19,1 & 49,7 & 55,8 \\
$1969-70$ & 46,2 & 20,7 & 37,9 & 19,1 & 50,9 & 57,6 \\
$1980-85$ & 41,3 & 19,1 & 36,9 & 18,4 & 55,6 & 62,6 \\
$1991-92$ & 42,2 & 21,8 & 39,5 & 21,2 & 58,4 & 64,2 \\
$2001-02$ & 44,9 & 26,2 & 41,6 & 25,4 & 60,7 & 66,7 \\
\hline
\end{tabular}

Fuente: Elaboración propia a base de Encuesta de Ocupación y desocupación del Gran Santiago, Universidad de Chile. Tablas de Mortalidad INE (2004).

Al estar calculados sobre la base de la prevalencia observada de las tasas de actividad e inactividad, los años brutos de vida activa reflejan las fluctuaciones en el tiempo de la participación en la fuerza de trabajo. En otras palabras, el Cuadro 3, nos seńala que la duración promedio de la vida o los años brutos de vida activa de un individuo tipo de una generación ficticia que experimentasen las mismas condiciones de actividad que en 1960-61 sería 42 años, mientras que en 2002 sería 45 años. Sin embargo, al tener en cuenta la mortalidad, los ańos netos vida activa, nos muestran el aumento real de años de vida activa gracias a los niveles más altos de sobrevivencia $\left(\mathrm{l}_{\mathrm{x}}\right)$ dentro de la PEA. ${ }^{16}$

Esperanza de Vida Activa de la fuerza de trabajo del Gran SANTIAGO I 960-2002

Desde las TVA obtenemos la "Esperanza de Vida Activa" - en adelante EVA- o "vida media potencialmente activa", la que tienen como principal virtud de condensar la estructura de inserción de la población en la actividad económica por edades, así como la incidencia de la mortalidad y las transiciones entre los estados de actividad e inactividad a lo largo de la vida activa de una cohorte ficticia. $^{17}$

De esta manera, al igual que sucede con la esperanza de vida, detrás del aumento de los años netos de vida activa están las continuas y significativas mejoras de las condiciones de salud y condiciones de vida de los trabajadores a lo largo del proceso de desarrollo social y económico, agregando años potenciales de trabajo de forma 
transversal a través de las distintas edades.

En este sentido, a excepción del periodo 1980-85 -fuertemente influenciado por ser un periodo marcado por la coyuntura de crisis en el caso de los hombres-, observamos que la EVA experimenta un aumento en todas las edades pero de mayor intensidad en las edades jóvenes -menores de 25 ańos-. En otras palabras, al inicio de la vida activa, en el periodo 1960-61 los hombres tenían 44,5 años de trabajo por delante y las mujeres, 36,2, mientras que en el periodo 2001-02 han aumentado a los 47,2 y 41,6 respectivamente. Por otro lado, a partir de los 50 ańos de edad se observa una disminución progresiva de la EVA. Este comportamiento en las edades avanzadas está acorde con el desarrollo del sistema de seguridad social (ver cuadro 4$){ }^{18}$

Cuadro 4. Gran Santiago: Esperanza de vida activa por grupo de edad y sexo

\begin{tabular}{|c|c|c|c|c|c|c|c|c|c|c|}
\hline \multicolumn{11}{|c|}{ Esperanza de vida activa } \\
\hline & \multicolumn{2}{|c|}{$1960-61$} & \multicolumn{2}{|c|}{$1969-70$} & \multicolumn{2}{|c|}{$1980-85$} & \multicolumn{2}{|c|}{ 1991-92 } & \multicolumn{2}{|c|}{ 2001-02 } \\
\hline & Hombre & Mujer & Hombre & Mujer & Hombre & Mujer & Hombre & Mujer & Hombre & Mujer \\
\hline 14 & 44,5 & 36,2 & 46,6 & 36,5 & 43,7 & 36,5 & 45,5 & 37,2 & 47,2 & 41,6 \\
\hline $15-19$ & 44,5 & 36,2 & 46,6 & 36,5 & 43,7 & 36,5 & 45,5 & 37,2 & 47,2 & 41,6 \\
\hline $20-24$ & 39,5 & 31,2 & 41,6 & 31,5 & 38,7 & 31,5 & 40,5 & 32,2 & 42,2 & 36,6 \\
\hline $25-29$ & 34,6 & 27,7 & 37,2 & 26,5 & 33,8 & 26,5 & 35,5 & 27,2 & 37,2 & 31,6 \\
\hline $30-34$ & 31,5 & 22,8 & 33,7 & 22,7 & 29,6 & 21,6 & 32,0 & 22,2 & 32,3 & 27,4 \\
\hline $35-39$ & 27,0 & 18,7 & 28,8 & 20,7 & 24,7 & 17,6 & 27,1 & 17,8 & 27,3 & 23,2 \\
\hline $40-44$ & 22,1 & 18,2 & 23,8 & 17,3 & 20,1 & 13,8 & 22,1 & 14,2 & 22,3 & 19,4 \\
\hline $45-49$ & 17,2 & 16,0 & 19,1 & 15,3 & 15,9 & 10,8 & 17,1 & 11,3 & 17,5 & 15,5 \\
\hline $50-54$ & 13,8 & 14,0 & 15,5 & 12,4 & 12,5 & 8,8 & 12,6 & 8,8 & 13,4 & 11,3 \\
\hline $55-59$ & 10,7 & 10,1 & 12,1 & 10,4 & 9,9 & 7,3 & 9,5 & 6,8 & 9,8 & 8,6 \\
\hline $60-64$ & 8,8 & 6,9 & 9,2 & 7,8 & 7,6 & 6,9 & 8,0 & 5,9 & 7,5 & 7,0 \\
\hline $65-69$ & 8,0 & 4,6 & 6,8 & 5,3 & 6,2 & 7,1 & 7,7 & 5,4 & 6,0 & 5,7 \\
\hline $70-74$ & 5,1 & 3,4 & 4,3 & 3,7 & 4,6 & 5,0 & 5,4 & 5,1 & 4,2 & 3,7 \\
\hline $75-79$ & 2,0 & 4,1 & 2,0 & 2,1 & 2,1 & 2,2 & 2,1 & 2,3 & 2,2 & 2,3 \\
\hline $80+$ & 0,0 & 0,0 & 0,0 & 0,0 & 0,0 & 0,0 & 0,0 & 0,0 & 0,0 & 0,0 \\
\hline
\end{tabular}

Fuente: Elaboración propia a base de Encuesta de Ocupación y desocupación del Gran Santiago, Universidad de Chile. Tablas de Mortalidad INE (2004).

Respecto de las brechas en la EVA entre hombres y mujeres estas son más abultadas en las edades de entrada a la actividad y disminuyen a medida que se acerca la edad de retiro. En perspectiva histórica, salvo en el periodo 1969-70 en la que la brecha de género 
aumenta, se puede observar una tendencia decreciente hacia el periodo 2001-02, siendo al inicio de la vida activa la mitad que treinta ańos antes.

\section{Comentarios finales}

En este artículo hemos querido plantear un aspecto fundamental de la fuerza de trabajo, la duración de la vida activa. Conocer el número promedio de años de actividad económica invertidos por los trabajadores es de invaluable utilidad tanto en la toma de decisiones para la planeación integral del desarrollo, como para la investigación académica. Por otro lado, con esta finalidad, hemos expuesto las contribuciones de una metodología práctica y sencilla para la elaboración de las TVA, que nos permiten analizar los cambios en las tasas netas y brutas de actividad así como en la EVA.

Como hemos podido observar, la fuerza de trabajo del Gran Santiago ha experimentado grandes transformaciones entre los periodos de 1960-61 y 2001-02. Estas transformaciones están motivadas por la combinación entre las dinámicas demográficas en la que destaca el avance de la esperanza de vida y las coyunturas económicas por las que el país atravesó dentro de este periodo. A través de las TVA, entre los cambios más importantes está el aumento de los años de EVA con que cada trabajador contribuye a la producción a lo largo de su vida. En este sentido, la reducción de la mortalidad, o en otras palabras el aumento de la esperanza de vida, actúa por un lado ha reducido los retiros anticipados de vida activa, y por otro, extiende el periodo de vida activa de los trabajadores, que incluso va más allá de los 65 años.

Si bien más años de vida activa pueden entenderse como un efecto positivo, este debe ir acompańado por la capacidad por parte del mercado de trabajo para absorber de forma productiva la creciente oferta de trabajo, de lo contrario, la a priori influencia positiva del retroceso de la mortalidad se transformará en un obstáculo al reemplazo generacional en el mercado de trabajo. En este sentido, la década de 1980 actúa como un punto de inflexión entre dos escenarios desde un punto de vista demográfico y económico, generando nuevas presiones en el mercado de trabajo.

Los estudios referidos a las características de la fuerza de trabajo, 
representan importantes avances para profundizar en investigaciones que vinculan las diferentes perspectivas que giran en torno del mercado de trabajo, provenientes de distintas ciencias sociales.

Bibliografía

ARELLANO, J. (1981). El efecto de las nuevas normas de la jubilación sobre el empleo. Estudios de Economía, 8(1), 135147.

CAMISA, Z. (1969), Argentina: aspectos demográcos de la población económicamente activa, 1974 y 1960-1980. Serie C 87, Centro Latinoamericano de Demografía, Santiago de Chile

CÁMARA, A. (2014). A biosocial approach to living conditions: intergenerational changes of stature dimorphism in 20thcentury Spain. Annals of Humman Biology, 42(2), 167-177.

CENTRO DE MICRODATOS (2016) Diseño muestral.

Encuesta de Ocupación y desocupación en el Gran Santiago. Departamento de Economía, Universidad de Chile, Santiago.

CERDA, R. A. (2007). Cambios demográficos: desafios y oportunidades de un nuevo escenario. Santiago de Chile: Pontificia Universidad Católica de Chile.

CHACKIEL, J. (2000). El envejecimieto de la población latinoamericana: ¿hacia una relación de dependencia favorable? Población y Desarrollo, num 4.

COWAN, K., MICCO, A., MIZALA, A., ROMAGUEDA, P., BRAVO, D., CERDA, R., y RAMOS, J. (2005). Un diagnóstico del desempleo en Chile. Universidad de Chile, Centro de Microdatos, Santiago de Chile.

DE RAMÓN, A. (1992). Santiago de Chile 1541-1991: Historia de una sociedad urbana. Santiago de Chile: Fundación MAPFRE.

DURAND, J. (1948). The labor force in United States, 18901960. New York: Social Science Research Council. 
DURAND, J. D. y A. MILLER (1969). Métodos de análisis de los datos censales relativos a las actividades económicas de la población. Estudios Demográficos num. 43, Naciones Unidas, Nueva York

ELIZAGA, J. (1970). Migraciones a las áreas metropolitanas de América Latina. Santiago de Chile: Centro Latinoamericano y Caribeño de Demografía (CELADE).

- (1979). Dinámica y economía de la población. Santiago de Chile: Centro Latinoamericano y Caribeño de Población (CELADE).

FUCARACCIO, A. (1974). El trabajo dela mujer en Chile en 1970: capital del país. Santiago de Chile: Centro Latinoamericano y Caribeño de Demografía .

GUTIÉRREZ DE MESA, J. (2010). Evolución de la actividad y el paro en Espańa y sus regiones en el periodo 20052010 a través de la esperanza de vida laboral en ausencia de mortalidad. $7^{a}$ Workshop-APDR. XXXVI reunión de Estudios Regionales-AECR. Badajoz-Elvas.

HAYWARD, M., y GRADY, W. (1990). Work and retirement amog a cohort of older men in the United States, 19661983. Demography, 27(3), 337-356.

HURTADO RUIZ-TAGLE, C. (1966). Concentración de la población y desarrollo económico. El caso de Chile. Santiago de Chile: Universidad de Chile.

LARRAÑAGA, O. (2006). Participación laboral de la mujer en Chile 1958-2003. Universidad de Chile, Departamento de Economía, Santiago de Chile.

MELlER, P. (1984). Análisis del problema de la elevada tasa de desocupación chilena. Santiago de Chile: Colección Estudios CIEPLAN.

MORELOS, J. (1996). Reseña de libro. Virgilio Partida Bush. La tabla de Vida Activa. Estudios Demográficos y Urbanos, 11(33), 647-651.

PAREDES, M. R., y González, M. P. (2002). Factores 
demográficos y la oferta de trabajo en Chile. El trimestre Económico, 69(4), 455-474.

PAREDES, R. (2003). Participación laboral de la mujer en ausencia de datos de panel: el caso de Chile. El Trimestre Económico, 70(279(3)), 407-422.

PARDO, L. (1988). Una revisión histórica a la participación de la población en la fuerza de trabajo. Tendencias y características de la participación de la mujer. Estudios de Economía, 15, 25-82.

PARTIDA-BUSH, V. (1996). Tabla de Vida Activa. México, D.F.: COLMEX.

(2000). Cambios en el mercado laboral urbano medidos a través de la esperanza de vida activa. Papeles de Población, 6(26).

_ (2014). Notas para un curso de demografía. México D.F.: Material de clase. FLACSO-México.

RINCÓN, M.(1977), Tablas de vida activa. Serie B 1008, Centro Latinoamericano de Demografía, Santiago de Chile.

RIVERO-CANTILLANO, R. (2016), El cambio demográfico en Chile y sus efectos sobre la fuerza de trabajo (1934-2006). Tesis de Doctorado. Universidad de Barcelona, España.

RIESCO, M. (2006). Tres exigencias mínimas para la reforma previsional. Documento En Foco 67, 67.

RIESCO, M. (2009). El modelo social chileno comienza a cambiar. Revista internacional del Trabajo, 128(3), 311-330. SOMOZA, J. (1970), Tablas de vida activa. Serie B 26, Centro Latinoamericano de Demografía, Santiago de Chile.

SULLIVAN, D. (1971). A single index of mortality and morbility. HSMHA Health Reports, 86(4), 347-354.

WOLFBEIN, S. L. (1949). The lenght of working life. Population Studies, 3(3), 286-294.

Fuentes

CENTRO DE MICRODATOS, Encuesta de Ocupación y 
Desocupación en el Gran Santiago. Departamento de Economía, Universidad de Chile. http://www.microdatos. $\mathrm{cl} /$ Encuestas/Ocupacion/OcupacionDesocupacion?C=T1 INE. (1952). XII Censo General de Población y I de vivienda. Santiago de Chile: Servicio Nacional de Estadística y Censos - Instituto ANcional de Estadísticas (INE).

- (1964). XIII Censo de Población y II de Vivienda (1960). Santiago de Chile: Dirección de Estadísitica y Censos Instituto Nacional de Estadísticas (INE) .

_ (1970). XIV Censo Nacional de Población y III de Vivienda 1970. Santiago de Chile: Dirección de Estadísticas y Censos - Instituto nacional de Estadísticas (INE).

_ (1982). XV Censo Nacional de Población y IV de Vivienda. Santiago de Chile: Dirección de Estadísticas y Censos Instituto nacional de Estadísticas (INE). (1992). XVI Censo Nacional de Población y V de Vivienda 1992. Santiago de Chile: Instituto Nacional de Estadísticas (INE). (2004). La mortalidad en Chile según las tablas abreviadas de mortalidad por sexo. Pais y Regiones, 1919-2002. Santiago de Chile: Instituto Naciona de Estadísticas (INE). 


\section{APÉNDICE}

Cuadro A.1. Gran Santiago: Probabilidades de transición
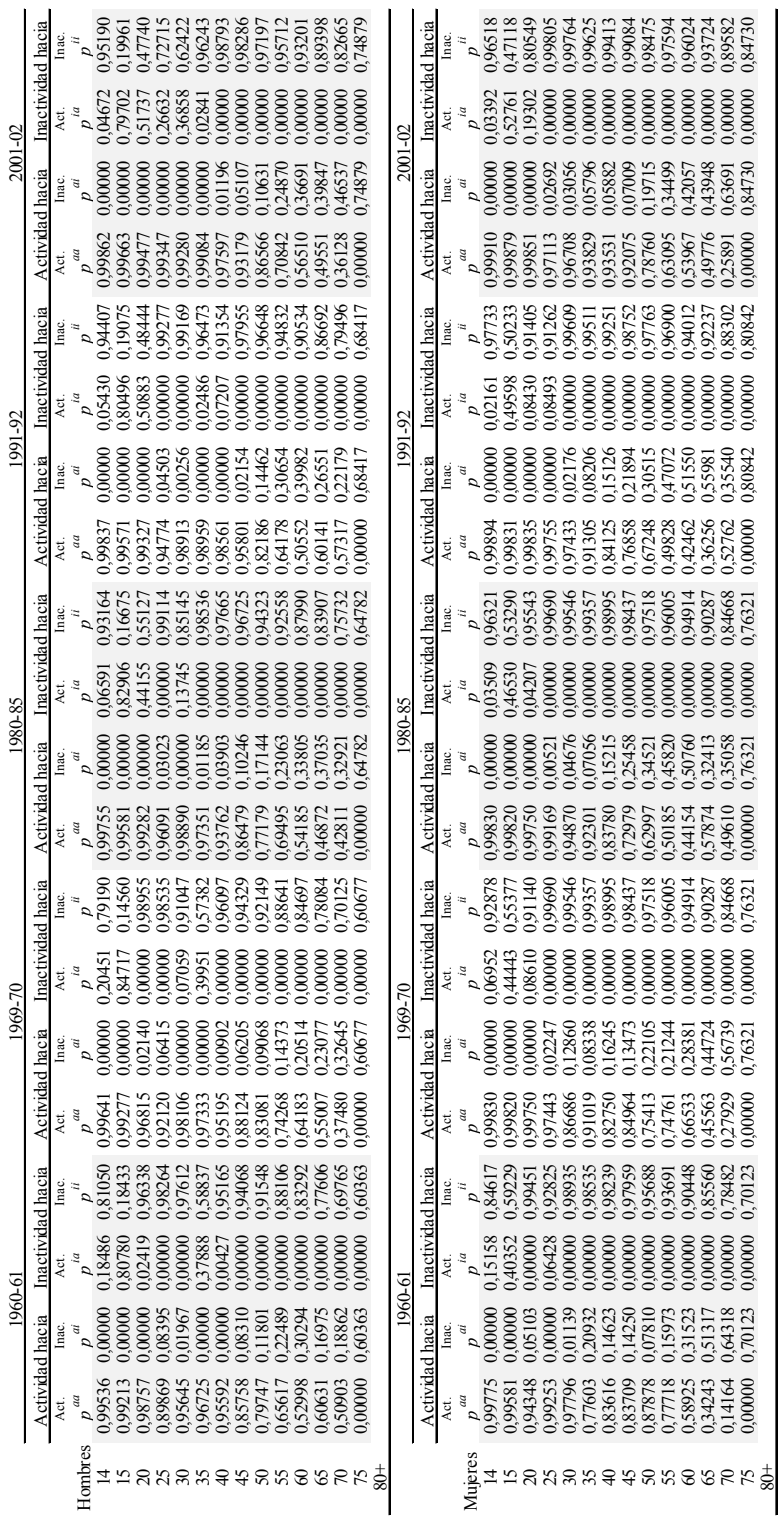

Fuente: Elaboración Propia a base de Encuesta de Ocupación y desocupación del Gran Santiago, Universidad de Chile. Tablas de Mortalidad INE (2004). 


\section{Cuadro A2. Gran Santiago: Tasas de ingreso y retiro de la fuerza de trabajo}

\begin{tabular}{|c|c|c|c|c|c|c|c|c|c|c|}
\hline & \multicolumn{10}{|c|}{ Ingresos } \\
\hline & \multicolumn{2}{|c|}{$1960-61$} & \multicolumn{2}{|c|}{$1969-70$} & \multicolumn{2}{|c|}{$1980-85$} & \multicolumn{2}{|c|}{$1991-92$} & \multicolumn{2}{|c|}{ 2001-02 } \\
\hline & Hombres & Mujeres & Hombres & Mujeres & Hombres & Mujeres & Hombres & Mujeres & Hombres & Mujeres \\
\hline & ${ }_{n} m^{i a}$ & ${ }_{n} m^{i a}$ & ${ }_{n} m^{i a}$ & ${ }_{n} m^{i a}$ & ${ }_{n} m^{i a}$ & ${ }_{n} m^{i a}$ & ${ }_{n} m^{i a}$ & ${ }_{n} m^{i a}$ & ${ }_{n} m^{i a}$ & ${ }_{n} m^{i a}$ \\
\hline 14 & 0,044 & 0,044 & 0,048 & 0,014 & 0,014 & 0,007 & 0,011 & 0,004 & 0,010 & 0,007 \\
\hline 15 & 0,212 & 0,100 & 0,271 & 0,107 & 0,202 & 0,105 & 0,194 & 0,132 & 0,190 & 0,120 \\
\hline 20 & 0,005 & 0,000 & 0,000 & 0,018 & 0,114 & 0,009 & 0,138 & 0,018 & 0,141 & 0,043 \\
\hline 25 & 0,000 & 0,000 & 0,000 & 0,000 & 0,000 & 0,000 & 0,000 & 0,018 & 0,054 & 0,000 \\
\hline 30 & 0,000 & 0,000 & 0,021 & 0,000 & 0,029 & 0,000 & 0,000 & 0,000 & 0,091 & 0,000 \\
\hline 35 & 0,097 & 0,000 & 0,103 & 0,000 & 0,000 & 0,000 & 0,005 & 0,000 & 0,006 & 0,000 \\
\hline \multirow[t]{5}{*}{40} & 0,001 & 0,000 & 0,000 & 0,000 & 0,000 & 0,000 & 0,018 & 0,000 & 0,000 & 0,000 \\
\hline & \multicolumn{10}{|c|}{ Muertes } \\
\hline & \multicolumn{2}{|c|}{$1960-61$} & \multicolumn{2}{|c|}{$1969-70$} & \multicolumn{2}{|c|}{$1980-85$} & \multicolumn{2}{|c|}{$1991-92$} & \multicolumn{2}{|c|}{ 2001-02 } \\
\hline & Hombres & Mujeres & Hombres & Mujeres & Hombres & Mujeres & Hombres & Mujeres & Hombres & Mujeres \\
\hline & ${ }^{n} m_{x}$ & ${ }^{n} \boldsymbol{m}_{x}$ & ${ }_{n} m_{x}$ & ${ }_{n} m_{x}$ & ${ }^{n} \boldsymbol{m}_{x}$ & ${ }^{n} m_{x}$ & ${ }^{n} m_{x}$ & ${ }^{n} m_{x}$ & ${ }_{n} m_{x}$ & ${ }_{n} \boldsymbol{m}_{x}$ \\
\hline 14 & 0,001 & 0,001 & 0,001 & 0,000 & 0,000 & 0,000 & 0,000 & 0,000 & 0,000 & 0,000 \\
\hline 15 & 0,002 & 0,001 & 0,001 & 0,001 & 0,001 & 0,000 & 0,001 & 0,000 & 0,001 & 0,000 \\
\hline 20 & 0,003 & 0,002 & 0,002 & 0,001 & 0,001 & 0,000 & 0,001 & 0,000 & 0,001 & 0,000 \\
\hline 25 & 0,004 & 0,002 & 0,003 & 0,001 & 0,002 & 0,001 & 0,001 & 0,000 & 0,001 & 0,000 \\
\hline 30 & 0,005 & 0,003 & 0,004 & 0,002 & 0,002 & 0,001 & 0,002 & 0,001 & 0,001 & 0,000 \\
\hline 35 & 0,007 & 0,004 & 0,005 & 0,003 & 0,003 & 0,001 & 0,002 & 0,001 & 0,002 & 0,001 \\
\hline 40 & 0,009 & 0,005 & 0,008 & 0,004 & 0,005 & 0,002 & 0,003 & 0,002 & 0,002 & 0,001 \\
\hline 45 & 0,012 & 0,007 & 0,012 & 0,004 & 0,007 & 0,003 & 0,004 & 0,003 & 0,003 & 0,002 \\
\hline 50 & 0,018 & 0,010 & 0,016 & 0,009 & 0,012 & 0,005 & 0,007 & 0,005 & 0,006 & 0,003 \\
\hline 55 & 0,025 & 0,016 & 0,024 & 0,013 & 0,015 & 0,008 & 0,011 & 0,006 & 0,009 & 0,005 \\
\hline 60 & 0,026 & 0,022 & 0,033 & 0,020 & 0,026 & 0,010 & 0,020 & 0,012 & 0,014 & 0,008 \\
\hline 65 & 0,050 & 0,034 & 0,049 & 0,031 & 0,035 & 0,020 & 0,029 & 0,016 & 0,022 & 0,013 \\
\hline 70 & 0,071 & 0,050 & 0,070 & 0,048 & 0,055 & 0,033 & 0,046 & 0,025 & 0,038 & 0,022 \\
\hline 75 & 0,099 & 0,076 & 0,098 & 0,070 & 0,085 & 0,054 & 0,075 & 0,042 & 0,057 & 0,033 \\
\hline \multirow[t]{5}{*}{$80+$} & 0,177 & 0,148 & 0,170 & 0,145 & 0,142 & 0,126 & 0,146 & 0,122 & 0,136 & 0,106 \\
\hline & \multicolumn{10}{|c|}{ Retiros } \\
\hline & \multicolumn{2}{|c|}{$1960-61$} & \multicolumn{2}{|c|}{$1969-70$} & \multicolumn{2}{|c|}{$1980-85$} & \multicolumn{2}{|c|}{$1991-92$} & \multicolumn{2}{|c|}{ 2001-02 } \\
\hline & Hombres & Mujeres & Hombres & Mujeres & Hombres & Mujeres & Hombres & Mujeres & Hombres & Mujeres \\
\hline & ${ }_{n} \boldsymbol{m}_{\boldsymbol{x}}$ & ${ }_{n} \boldsymbol{m}_{x}$ & ${ }_{n} \boldsymbol{m}_{\boldsymbol{x}}$ & ${ }_{n} \boldsymbol{m}_{x}$ & ${ }_{n} \boldsymbol{m}_{x}$ & ${ }_{n} \boldsymbol{m}_{x}$ & ${ }_{n} \boldsymbol{m}_{x}$ & ${ }_{n} \boldsymbol{m}_{x}$ & ${ }_{n} \boldsymbol{m}_{x}$ & ${ }_{n} \boldsymbol{m}_{x}$ \\
\hline 20 & 0,000 & 0,011 & 0,004 & 0,000 & 0,000 & 0,000 & 0,000 & 0,000 & 0,000 & 0,000 \\
\hline 25 & 0,017 & 0,000 & 0,013 & 0,005 & 0,006 & 0,001 & 0,009 & 0,000 & 0,000 & 0,005 \\
\hline 30 & 0,004 & 0,003 & 0,000 & 0,028 & 0,000 & 0,010 & 0,001 & 0,005 & 0,000 & 0,006 \\
\hline 35 & 0,000 & 0,048 & 0,000 & 0,017 & 0,003 & 0,015 & 0,000 & 0,017 & 0,000 & 0,012 \\
\hline 40 & 0,000 & 0,081 & 0,002 & 0,036 & 0,008 & 0,033 & 0,000 & 0,033 & 0,002 & 0,012 \\
\hline 45 & 0,018 & 0,061 & 0,014 & 0,029 & 0,022 & 0,059 & 0,005 & 0,050 & 0,011 & 0,014 \\
\hline 50 & 0,028 & 0,017 & 0,021 & 0,051 & 0,040 & 0,086 & 0,032 & 0,074 & 0,023 & 0,044 \\
\hline 55 & 0,059 & 0,036 & 0,035 & 0,050 & 0,057 & 0,125 & 0,077 & 0,128 & 0,060 & 0,086 \\
\hline 60 & 0,099 & 0,084 & 0,055 & 0,070 & 0,095 & 0,146 & 0,113 & 0,151 & 0,098 & 0,112 \\
\hline 65 & 0,049 & 0,171 & 0,069 & 0,132 & 0,113 & 0,088 & 0,072 & 0,174 & 0,115 & 0,123 \\
\hline 70 & 0,063 & 0,278 & 0,121 & 0,202 & 0,111 & 0,104 & 0,065 & 0,101 & 0,157 & 0,221 \\
\hline 75 & 0,400 & 0,170 & 0,400 & 0,400 & 0,400 & 0,400 & 0,400 & 0,400 & 0,400 & 0,400 \\
\hline $80+$ & 0,000 & 0,000 & 0,000 & 0,000 & 0,000 & 0,000 & 0,000 & 0,000 & 0,000 & 0,000 \\
\hline
\end{tabular}

Fuente: Elaboración Propia a base de Encuesta de Ocupación y desocupación del Gran Santiago, Universidad de Chile. Tablas de Mortalidad INE (2004). 
Cuadro A.3. Gran Santiago: Años brutos y netos de vida activa e inactiva

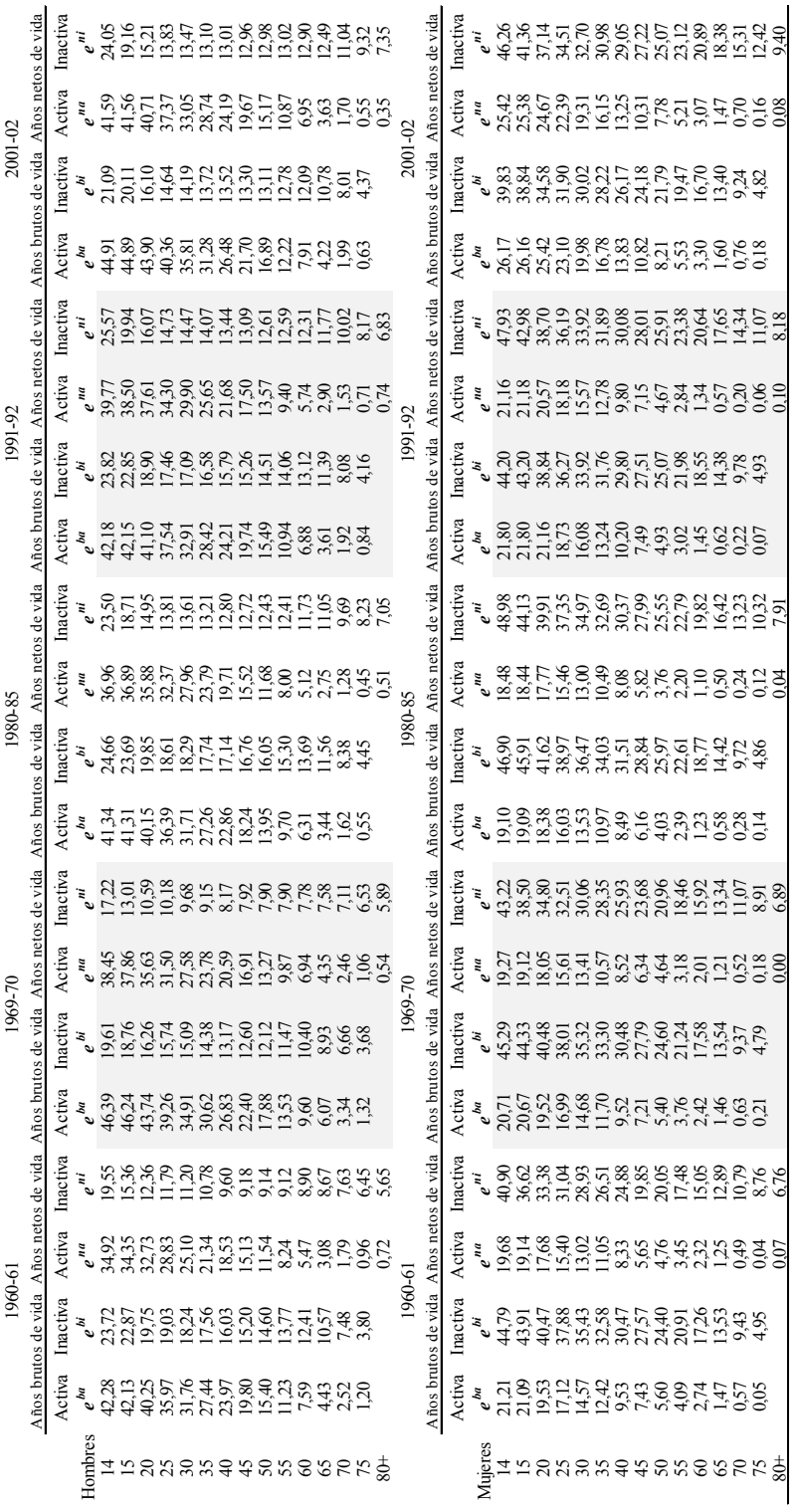

Fuente: Elaboración Propia a base de EOD del Gran Santiago, Universidad de Chile. Tablas de Mortalidad INE (2004). 
Cuadro A4. Gran Santiago: Tabla abreviada de vida activa

\begin{tabular}{|c|c|c|c|c|c|c|c|c|c|}
\hline \multicolumn{10}{|c|}{$\begin{array}{c}1960-61 \\
\text { Hombres }\end{array}$} \\
\hline$x$ & $n$ & $\mathrm{I}_{\mathrm{x}}$ & ${ }_{n} L_{x}$ & ${ }_{n} L^{a}$ & $\mathrm{~T}_{\mathrm{x}}$ & $\mathrm{T}^{\mathrm{a}}$ & $e_{x}$ & $e^{\text {na }}$ & $e^{a a}$ \\
\hline 14 & 1 & 87.554 & 436.755 & 64.446 & 4.769.110 & 3.057 .783 & 54,47 & 34,92 & 44,51 \\
\hline 15 & 5 & 87.148 & 434.024 & 163.433 & 4.332.355 & 2.993 .337 & 49,71 & 34,35 & 44,51 \\
\hline 20 & 5 & 86.462 & 429.623 & 367.894 & 3.898 .331 & 2.829 .903 & 45,09 & 32,73 & 39,53 \\
\hline 25 & 5 & 85.387 & 423.231 & 355.986 & 3.468 .708 & 2.462 .009 & 40,62 & 28,83 & 34,57 \\
\hline 30 & 5 & 83.905 & 414.515 & 358.029 & 3.045 .477 & 2.106 .023 & 36,3 & 25,10 & 31,54 \\
\hline 35 & 5 & 81.901 & 402.801 & 280.137 & 2.630 .962 & 1.747 .994 & 32,12 & 21,34 & 27,00 \\
\hline 40 & 5 & 79.219 & 387.366 & 322.409 & 2.228 .161 & 1.467 .857 & 28,13 & 18,53 & 22,08 \\
\hline 45 & 5 & 75.727 & 367.406 & 323.480 & 1.840 .795 & 1. 145.448 & 24,31 & 15,13 & 17,19 \\
\hline 50 & 5 & 71.235 & 341.124 & 284.359 & 1.473 .389 & 821.968 & 20,68 & 11,54 & 13,77 \\
\hline 55 & 5 & 65.214 & 306.679 & 223.574 & 1.132 .265 & 537.609 & 17,36 & 8,24 & 10,67 \\
\hline 60 & 5 & 57.457 & 263.287 & 166.443 & 825.586 & 314.035 & 14,37 & 5,47 & 8,82 \\
\hline 65 & 5 & 47.857 & 212.495 & 81.081 & 562.299 & 147.592 & 11,75 & 3,08 & 7,96 \\
\hline 70 & 5 & 37.140 & 157.628 & 41.531 & 349.804 & 66.511 & 9,42 & 1,79 & 5,13 \\
\hline 75 & 5 & 25.911 & 103.879 & 24.980 & 192.176 & 24.980 & 7,42 & 0,96 & 2,00 \\
\hline $80+$ & 0 & 15.640 & 88.297 & 11.233 & 99.530 & 11.233 & 5,65 & 0,72 & 0,00 \\
\hline \multicolumn{10}{|c|}{ Mujeres } \\
\hline$x$ & $\mathrm{n}$ & $\mathrm{I}_{\mathrm{x}}$ & $n L_{x}$ & ${ }_{n} L^{a}$ & $\mathrm{~T}_{\mathrm{x}}$ & $\mathrm{T}^{\mathrm{a}}$ & $e_{x}$ & $e^{\text {na }}$ & $e^{a a}$ \\
\hline 14 & 1 & 89.146 & 444.986 & 53.266 & 5.399 .794 & 1.754 .091 & 60,57 & 19,68 & 44,51 \\
\hline 15 & 5 & 88.848 & 443.033 & 138.680 & 4.954 .808 & 1.700 .825 & 55,77 & 19,14 & 44,51 \\
\hline 20 & 5 & 88.365 & 440.065 & 211.794 & 4.511 .775 & 1.562 .145 & 51,06 & 17,68 & 39,53 \\
\hline 25 & 5 & 87.661 & 435.830 & 222.224 & 4.071 .710 & 1.350 .351 & 46,45 & 15,40 & 34,57 \\
\hline 30 & 5 & 86.671 & 430.106 & 185.128 & 3.635 .880 & 1.128 .127 & 41,95 & 13,02 & 31,54 \\
\hline 35 & 5 & 85.371 & 422.910 & 244.662 & 3.205 .774 & 942.998 & 37,55 & 11,05 & 27,00 \\
\hline 40 & 5 & 83.793 & 414.145 & 173.448 & 2.782 .864 & 698.337 & 33,21 & 8,33 & 22,08 \\
\hline 45 & 5 & 92.865 & 402.774 & 147.677 & 2.368 .719 & 524.889 & 28,93 & 5,65 & 17,19 \\
\hline 50 & 5 & 79.244 & 386.729 & 116.751 & 1.965 .945 & 377.212 & 24,81 & 4,76 & 13,77 \\
\hline 55 & 5 & 75.448 & 363.569 & 97.946 & 1.579 .216 & 260.461 & 20,93 & 3,45 & 10,67 \\
\hline 60 & 5 & 69.980 & 331.364 & 84.586 & 1.215 .647 & 162.515 & 17,37 & 2,32 & 8,82 \\
\hline 65 & 5 & 62.566 & 288.490 & 51.914 & 884.283 & 77.928 & 14,13 & 1,25 & 7,96 \\
\hline 70 & 5 & 52.830 & 234.680 & 24.310 & 595.793 & 26.014 & 11,28 & 0,49 & 5,13 \\
\hline 75 & 5 & 41.042 & 172.367 & 1.705 & 361.113 & 1.705 & 8,8 & 0,04 & 2,00 \\
\hline $80+$ & 0 & 27.905 & 188.746 & 1.999 & 190.745 & 1.999 & 6,76 & 0,07 & 0,00 \\
\hline
\end{tabular}

Fuente: Elaboración Propia a base de Encuesta de Ocupación y desocupación del Gran Santiago, Universidad de Chile. Tablas de Mortalidad INE (2004). 


\begin{tabular}{|c|c|c|c|c|c|c|c|c|c|}
\hline \multicolumn{10}{|c|}{$\begin{array}{l}1969-70 \\
\text { Hombres }\end{array}$} \\
\hline$x$ & $n$ & $I_{x}$ & ${ }_{n} L_{x}$ & ${ }_{n} L^{a}$ & $T_{x}$ & $\mathrm{~T}^{\mathrm{a}}$ & $e_{x}$ & $e^{\text {na }}$ & $e^{a a}$ \\
\hline 14 & 1 & 92.357 & 460.955 & 67.512 & 5.141 .622 & 3.551 .311 & 55,67 & 38,45 & 46,57 \\
\hline 15 & 5 & 92.025 & 458.463 & 228.848 & 4.680 .667 & 3.483 .799 & 50,86 & 37,86 & 46,57 \\
\hline 20 & 5 & 91.360 & 454.413 & 407.487 & 4.222 .204 & 3.254 .951 & 46,21 & 35,63 & 41,59 \\
\hline 25 & 5 & 90.405 & 448.715 & 390.452 & 3.767 .791 & 2.847 .464 & 41,68 & 31,50 & 37,18 \\
\hline 30 & 5 & 89.081 & 441.188 & 378.767 & 3.319 .076 & 2.457 .012 & 37,26 & 27,58 & 33,70 \\
\hline 35 & 5 & 87.394 & 431.143 & 326.636 & 2.877 .888 & 2.078 .245 & 32,93 & 23,78 & 28,75 \\
\hline 40 & 5 & 85.063 & 417.015 & 369.533 & 2.446 .745 & 1.751 .608 & 28,76 & 20,59 & 23,82 \\
\hline 45 & 5 & 81.743 & 397.125 & 358.935 & 2.029 .730 & 1.382 .076 & 24,83 & 16,91 & 19,14 \\
\hline 50 & 5 & 77.107 & 370.400 & 322.005 & 1.632 .605 & 1.023 .140 & 21,17 & 13,27 & 15,46 \\
\hline 55 & 5 & 71.053 & 335.088 & 263.779 & 1.262 .205 & 701.136 & 17,76 & 9,87 & 12,08 \\
\hline 60 & 5 & 62.982 & 290.815 & 205.413 & 927.117 & 437.356 & 14,72 & 6,94 & 9,25 \\
\hline 65 & 5 & 53.344 & 237.493 & 129.439 & 636.302 & 231.943 & 11,93 & 4,35 & 6,85 \\
\hline 70 & 5 & 41.653 & 177.155 & 71.438 & 398.809 & 102.503 & 9,57 & 2,46 & 4,34 \\
\hline 75 & 5 & 29.209 & 117.330 & 31.065 & 221.654 & 31.065 & 7,59 & 1,06 & 2,01 \\
\hline $80+$ & 0 & 17.723 & 104.324 & 9.626 & 113.950 & 9.626 & 5,89 & 0,54 & 0,00 \\
\hline \multicolumn{10}{|c|}{ Mujeres } \\
\hline$x$ & $\mathrm{n}$ & $I_{x}$ & ${ }_{n} L_{x}$ & ${ }_{n} L^{a}$ & $\mathrm{~T}_{\mathrm{x}}$ & $\mathrm{T}^{\mathrm{a}}$ & $e_{x}$ & $e^{\text {na }}$ & $e^{a a}$ \\
\hline 14 & 1 & 93.917 & 469.058 & 17.648 & 5.868 .112 & 1.809 .480 & 62,48 & 19,27 & 36,49 \\
\hline 15 & 5 & 93.706 & 467.548 & 107.398 & 5.399 .054 & 1.791 .831 & 57,62 & 19,12 & 36,49 \\
\hline 20 & 5 & 93.313 & 465.285 & 235.568 & 4.931 .506 & 1.684 .433 & 52,85 & 18,05 & 31,50 \\
\hline 25 & 5 & 92.801 & 462.273 & 213.311 & 4.466 .221 & 1.448 .865 & 48,13 & 15,61 & 26,51 \\
\hline 30 & 5 & 92.108 & 458.088 & 272.743 & 4.003 .948 & 1.235 .554 & 43,47 & 13,41 & 22,68 \\
\hline 35 & 5 & 91.127 & 452.298 & 197.936 & 3.545 .860 & 962.811 & 38,91 & 10,57 & 20,70 \\
\hline 40 & 5 & 89.792 & 445.008 & 205.570 & 3.093 .562 & 764.876 & 34,45 & 8,52 & 17,32 \\
\hline 45 & 5 & 88.211 & 436.555 & 158.091 & 2.648 .554 & 559.306 & 30,03 & 6,34 & 15,28 \\
\hline 50 & 5 & 86.411 & 422.740 & 138.117 & 2.211 .999 & 401.215 & 25,6 & 4,64 & 12,36 \\
\hline 55 & 5 & 82.685 & 400.383 & 107.243 & 1.789 .259 & 263.098 & 21,64 & 3,18 & 10,38 \\
\hline 60 & 5 & 77.468 & 368.840 & 70.796 & 1.388 .876 & 155.855 & 17,93 & 2,01 & 7,79 \\
\hline 65 & 5 & 70.068 & 325.045 & 53.993 & 1.020 .036 & 85.059 & 41,56 & 1,21 & 5,34 \\
\hline 70 & 5 & 59.950 & 267.500 & 22.705 & 694.991 & 31.066 & 11,59 & 0,52 & 3,67 \\
\hline 75 & 5 & 47.050 & 200.108 & 8.361 & 427.491 & 8.361 & 9,09 & 0,18 & 2,13 \\
\hline $80+$ & 0 & 32.993 & 227.383 & 0 & 227.383 & 0 & 6,89 & 0,00 & 0,00 \\
\hline
\end{tabular}

Fuente: Elaboración Propia a base de Encuesta de Ocupación y desocupación del Gran Santiago, Universidad de Chile. Tablas de Mortalidad INE (2004). 


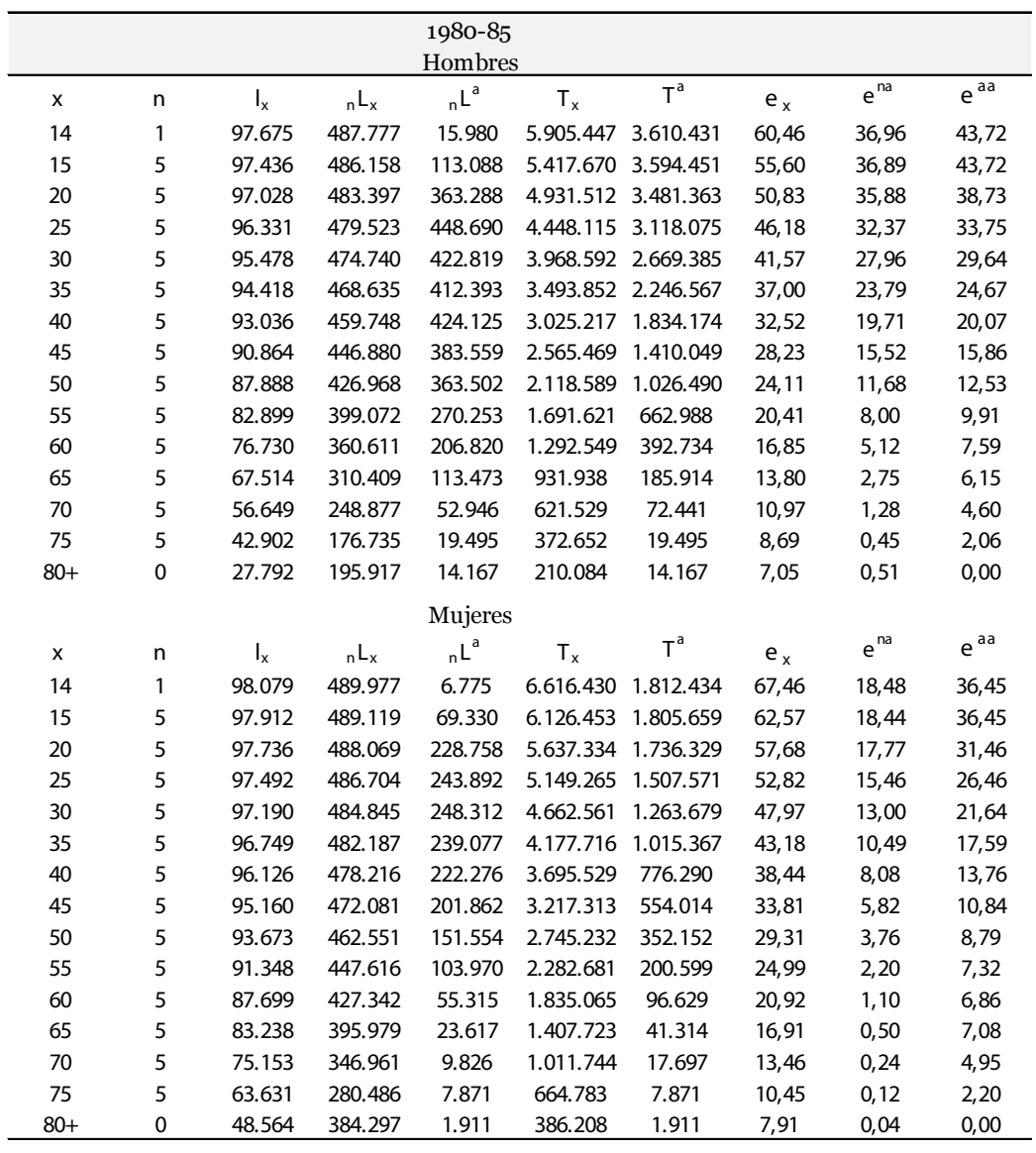

Fuente: Elaboración Propia a base de Encuesta de Ocupación y desocupación del Gran Santiago, Universidad de Chile. Tablas de Mortalidad INE (2004). 


\begin{tabular}{|c|c|c|c|c|c|c|c|c|c|}
\hline \multicolumn{10}{|c|}{$\begin{array}{l}\text { 1991-92 } \\
\text { Hombres }\end{array}$} \\
\hline$x$ & $\mathrm{n}$ & $\mathrm{I}_{\mathrm{x}}$ & ${ }_{n} L_{x}$ & ${ }_{n} L^{a}$ & $\mathrm{~T}_{\mathrm{x}}$ & $T^{a}$ & $e_{x}$ & $e^{\text {na }}$ & $\mathrm{e}^{\mathrm{aa}}$ \\
\hline 14 & 1 & 95.254 & 490.868 & 11.277 & 6.223 .618 & 3.788 .383 & 63,34 & 39,77 & 45,49 \\
\hline 15 & 5 & 98.094 & 489.416 & 103.500 & 5.732 .750 & 3.777 .106 & 58,44 & 38,50 & 45,49 \\
\hline 20 & 5 & 97.673 & 486.720 & 346.243 & 5.243 .334 & 3.673 .606 & 53,68 & 37,61 & 40,50 \\
\hline 25 & 5 & 97.015 & 483.324 & 447.706 & 4.756 .614 & 3.327 .363 & 49,03 & 34,30 & 35,51 \\
\hline 30 & 5 & 96.314 & 479.569 & 430.166 & 4.273 .290 & 2.879 .657 & 44,97 & 29,90 & 31,98 \\
\hline 35 & 5 & 95.514 & 475.082 & 400.782 & 3.793.721 & 2.449 .490 & 39,72 & 25,65 & 27,07 \\
\hline 40 & 5 & 94.519 & 469.196 & 418.755 & 3.318 .639 & 2.048 .709 & 35,11 & 21,68 & 22,09 \\
\hline 45 & 5 & 93.159 & 461.033 & 391.987 & 2.849 .443 & 1.629 .954 & 30,58 & 17,50 & 17,13 \\
\hline 50 & 5 & 91.254 & 448.623 & 408.833 & 2.388 .410 & 1.237 .967 & 26,17 & 13,57 & 12,63 \\
\hline 55 & 5 & 88.195 & 429.851 & 348.823 & 1.939 .787 & 829.134 & 21,99 & 9,40 & 9,50 \\
\hline 60 & 5 & 83.637 & 398.394 & 260.353 & 1.509 .936 & 480.311 & 18,05 & 5,74 & 8,00 \\
\hline 65 & 5 & 75.720 & 353.409 & 119.434 & 1.111 .542 & 219.958 & 14,68 & 2,90 & 7,68 \\
\hline 70 & 5 & 65.643 & 294.568 & 63.524 & 758.133 & 100.524 & 11,55 & 1,53 & 5,38 \\
\hline 75 & 5 & 52.184 & 219.716 & 37.000 & 463.565 & 37.000 & 8,88 & 0,71 & 2,11 \\
\hline $80+$ & 0 & 35.703 & 243.849 & 26.459 & 270.308 & 26.459 & 6,83 & 0,74 & 0,00 \\
\hline \multicolumn{10}{|c|}{ Mujeres } \\
\hline$x$ & $\mathrm{n}$ & $\mathrm{I}_{\mathrm{x}}$ & ${ }_{n} L_{x}$ & ${ }_{n} L^{a}$ & $\mathrm{~T}_{\mathrm{x}}$ & $T^{a}$ & $e_{x}$ & $e^{n a}$ & $\mathrm{e}^{\mathrm{aa}}$ \\
\hline 14 & 1 & 98.611 & 492.796 & 0 & 6.813 .246 & 2.086 .820 & 69,09 & 21,16 & 37,21 \\
\hline 15 & 5 & 98.507 & 492.118 & 63.874 & 6.320 .450 & 2.086 .820 & 64,16 & 21,18 & 37,21 \\
\hline 20 & 5 & 98.340 & 491.296 & 238.520 & 5.828 .332 & 2.022 .946 & 59,27 & 20,57 & 32,22 \\
\hline 25 & 5 & 98.178 & 490.289 & 259.815 & 5.337 .036 & 1.784 .425 & 54,36 & 18,18 & 27,22 \\
\hline 30 & 5 & 97.938 & 488.731 & 277.909 & 4.846 .747 & 1.524 .610 & 46,46 & 15,57 & 22,23 \\
\hline 35 & 5 & 97.555 & 486.581 & 295.192 & 4.358 .016 & 1.246.701 & 44,67 & 12,78 & 17,79 \\
\hline 40 & 5 & 97.078 & 483.570 & 262.559 & 3.871 .435 & 951.509 & 39,88 & 9,80 & 14,18 \\
\hline 45 & 5 & 96.351 & 478.746 & 245.051 & 3.387 .865 & 688.951 & 35,16 & 7,15 & 11,30 \\
\hline 50 & 5 & 95.148 & 470.419 & 179.795 & 2.909 .119 & 443.899 & 30,57 & 4,67 & 8,84 \\
\hline 55 & 5 & 93.020 & 457.889 & 143.613 & 2.438 .700 & 264.104 & 26,22 & 2,84 & 6,78 \\
\hline 60 & 5 & 90.136 & 437.187 & 72.139 & 1.980 .811 & 120.492 & 21,98 & 1,34 & 5,94 \\
\hline 65 & 5 & 84.739 & 407.248 & 33.038 & 1.543 .624 & 48.353 & 18,22 & 0,57 & 5,36 \\
\hline 70 & 5 & 78.160 & 367.944 & 11.055 & 1.136 .376 & 15.315 & 15,54 & 0,20 & 5,11 \\
\hline 75 & 5 & 69.017 & 312.030 & 4.260 & 768.432 & 4.260 & 11,13 & 0,06 & 2,26 \\
\hline $80+$ & 0 & 55.795 & 456.402 & 5.685 & 462.087 & 5.685 & 8,18 & 0,10 & 0,00 \\
\hline
\end{tabular}

Fuente: Elaboración Propia a base de Encuesta de Ocupación y desocupación del Gran Santiago, Universidad de Chile. Tablas de Mortalidad INE (2004). 


\begin{tabular}{|c|c|c|c|c|c|c|c|c|c|}
\hline \multicolumn{10}{|c|}{$\begin{array}{l}\text { 2001-02 } \\
\text { Hombres }\end{array}$} \\
\hline$x$ & $\mathrm{n}$ & $\mathrm{I}_{\mathrm{x}}$ & ${ }_{n} L_{x}$ & ${ }_{n} L^{a}$ & $\mathrm{~T}_{\mathrm{x}}$ & $\mathrm{T}^{\mathrm{a}}$ & $e_{x}$ & $e^{\text {na }}$ & $\mathrm{e}^{\mathrm{aa}}$ \\
\hline 14 & 1 & 98.951 & 494.415 & 8.166 & 6.494 .801 & 4.115 .045 & 65,64 & 41,59 & 47,22 \\
\hline 15 & 5 & 98.815 & 493.242 & 97.714 & 6.000 .386 & 4.106.879 & 60,72 & 41,56 & 47,22 \\
\hline 20 & 5 & 98.482 & 491.123 & 348.042 & 5.507 .144 & 4.009 .165 & 55,92 & 40,71 & 42,23 \\
\hline 25 & 5 & 97.967 & 488.237 & 444.054 & 5.016 .021 & 3.661 .124 & 51,20 & 37,37 & 37,24 \\
\hline 30 & 5 & 97.328 & 484.886 & 439.912 & 4.527 .784 & 3.217 .069 & 46,52 & 33,05 & 32,26 \\
\hline 35 & 5 & 96.627 & 480.920 & 461.023 & 4.042 .898 & 2.777 .157 & 41,84 & 28,74 & 27,27 \\
\hline 40 & 5 & 95.741 & 475.817 & 455.377 & 3.561 .978 & 2.316 .134 & 37,20 & 24,19 & 22,30 \\
\hline 45 & 5 & 94.585 & 468.875 & 450.610 & 3.086.161 & 1.860 .757 & 32,63 & 19,67 & 17,55 \\
\hline 50 & 5 & 92.965 & 458.309 & 427.980 & 2.617 .286 & 1.410 .147 & 28,15 & 15,17 & 13,42 \\
\hline 55 & 5 & 90.359 & 442.109 & 381.360 & 2.158.977 & 982.166 & 23,89 & 10,87 & 9,83 \\
\hline 60 & 5 & 86.485 & 417.722 & 308.007 & 1.716 .868 & 600.806 & 19,85 & 6,95 & 7,53 \\
\hline 65 & 5 & 80.604 & 381.657 & 170.545 & 1.299 .146 & 292.799 & 16,12 & 3,63 & 6,03 \\
\hline 70 & 5 & 72.059 & 329.065 & 89.229 & 917.489 & 122.254 & 12,73 & 1,70 & 4,24 \\
\hline 75 & 5 & 59.567 & 260.426 & 33.026 & 588.424 & 33.026 & 9,88 & 0,55 & 2,19 \\
\hline $80+$ & 0 & 44.603 & 327.998 & 15.454 & 343.452 & 15.454 & 7,35 & 0,35 & 0,00 \\
\hline \multicolumn{10}{|c|}{ Mujeres } \\
\hline$x$ & $\mathrm{n}$ & $I_{x}$ & ${ }_{n} L_{x}$ & ${ }_{n} L^{a}$ & $\mathrm{~T}_{\mathrm{x}}$ & $\mathrm{T}^{\mathrm{a}}$ & $e_{x}$ & $e^{n a}$ & $\mathrm{e}^{\mathrm{aa}}$ \\
\hline 14 & 1 & 99.133 & 495.443 & 5.523 & 7.105.435 & 2.519 .470 & 71,68 & 25,42 & 41,60 \\
\hline 15 & 5 & 99.044 & 494.921 & 73.232 & 6.609 .992 & 2.513 .947 & 66,74 & 25,38 & 41,60 \\
\hline 20 & 5 & 98.925 & 494.254 & 229.035 & 6.115 .071 & 2.440 .716 & 61,82 & 24,67 & 36,60 \\
\hline 25 & 5 & 98.777 & 493.405 & 308.444 & 5.620 .817 & 2.211 .681 & 56,9 & 22,39 & 31,60 \\
\hline 30 & 5 & 98.585 & 492.341 & 314.895 & 5.127 .412 & 1.903 .237 & 52,01 & 19,31 & 27,41 \\
\hline 35 & 5 & 98.352 & 490.837 & 290.092 & 4.635.071 & 1.588 .342 & 47,13 & 16,15 & 23,20 \\
\hline 40 & 5 & 97.983 & 488.478 & 294.009 & 4.144.234 & 1.298 .250 & 42,3 & 13,25 & 19,38 \\
\hline 45 & 5 & 97.408 & 484.810 & 252.882 & 3.655 .756 & 1.004 .241 & 37,53 & 10,31 & 15,46 \\
\hline 50 & 5 & 96.516 & 478.898 & 256.410 & 3.170 .946 & 751.359 & 32,85 & 7,78 & 11,35 \\
\hline 55 & 5 & 95.044 & 469.501 & 209.791 & 2.692 .048 & 494.949 & 28,32 & 5,21 & 8,61 \\
\hline 60 & 5 & 92.757 & 454.563 & 153.993 & 2.222 .547 & 285.158 & 23,96 & 3,07 & 7,02 \\
\hline 65 & 5 & 89.069 & 431.368 & 72.959 & 1.767 .984 & 131.165 & 19,85 & 1,47 & 5,68 \\
\hline 70 & 5 & 83.479 & 395.650 & 46.022 & 1.336.616 & 58.207 & 16,01 & 0,70 & 3,72 \\
\hline 75 & 5 & 74.782 & 345.360 & 12.185 & 940.966 & 12.185 & 12,58 & 0,16 & 2,31 \\
\hline $80+$ & 0 & 63.362 & 595.606 & 5.083 & 600.689 & 5.083 & 9,4 & 0,08 & 0,00 \\
\hline
\end{tabular}

Fuente: Elaboración Propia a base de Encuesta de Ocupación y desocupación del Gran Santiago, Universidad de Chile. Tablas de Mortalidad INE (2004). 


\section{Apéndice metodológico: La tabla de Vida aCtiva}

- Años brutos y netos de vida activa:

La duración de la vida activa depende del efecto de la mortalidad y los niveles de participación de la población en edades económicamente activa, ambos efectos pueden variar según características de grupos de población como la edad o el sexo. Dos medidas de duración de la vida activa son los "años brutos" y "años netos" de vida activa. Los años brutos de vida activa representan el número medio de años de actividad de una cohorte en ausencia de mortalidad. Mientras que los años netos de vida activa toma en cuenta las salidas por muerte (Elizaga, 1979; Partida-Bush, 1996; 2014).

Un paso previo es calcular las proporciones de actividad ${ }_{\mathrm{n}} A_{\mathrm{x}} \mathrm{e}$ inactividad $\underset{\mathrm{n} x}{I}$

$$
\begin{gathered}
A_{\mathrm{n}}=\frac{P E A_{\mathrm{x}}}{P_{\mathrm{x}}} \\
I_{\mathrm{n}}=\frac{{ }_{\mathrm{n}} P E I_{\mathrm{x}}}{P_{\mathrm{x}}}
\end{gathered}
$$

Donde PEA es la población activa a la edad " $x$ " en el intervalo de tiempo " $n$ ". Y de igual modo PEI corresponde a la población inactiva a edad " $x$ " en el intervalo de tiempo " $n$ ". Cuyo denominador es la población a la edad " $\mathrm{x}$ " en el intervalo de tiempo " $\mathrm{n}$ ".

Los años brutos de vida activa $\left(e^{b a}\right)$ los calculamos de la siguiente manera:

$$
e^{b a}=\sum_{y=x}^{\theta-n} \mathrm{n}_{n} a_{x}
$$

Y los años brutos de vida inactiva $\left(e^{b i}\right)$ :

$$
e^{b i}=\sum_{y=x}^{\theta-n} \mathrm{n}_{n}^{i}
$$

Donde $\mathrm{n}_{\mathrm{n}} A_{\mathrm{x}}$ corresponde a la proporción de la actividad multiplicado por el intervalo.

Si tenemos en cuenta el riesgo de morir obtenemos los años netos de vida activa e inactiva $\left(\mathrm{e}^{\mathrm{na}} \mathrm{y} \mathrm{e}^{\mathrm{ni}}\right)$. Para esto tomamos los sobrevivientes $\left(l_{\mathrm{x}}\right)$ y los años-persona $\left({ }_{\mathrm{n}} L_{\mathrm{x}}\right)$ de la tabla de mortalidad (INE, 2004).

De este modo los ańos netos de vida activa e inactiva son: 


$$
e^{n a}=\frac{T_{x}^{a}}{l x} \quad \text { y } \quad e^{n i}=\frac{T_{x}^{i}}{l x}
$$

Donde $T_{x}^{a}$ y $T_{x}^{i}$ corresponden a los ańos-persona vividos en actividad e inactividad a la edad $x$ :

$$
\begin{aligned}
& T_{x}^{a}=\sum_{y=x}^{\theta-n}{ }_{n} L_{y}^{a} \\
& T_{x}^{i}=\sum_{y=x}^{\theta-n} L_{y}^{i}
\end{aligned}
$$

El tiempo vivido en actividad e inactividad en el intervalo es:

$$
{ }_{n} L_{x}^{a}={ }_{n} L_{x} A_{x} \quad \text { y }{ }_{n} L_{x}^{i}={ }_{n} L_{x n} I_{x}
$$

En el caso del tiempo vivido en actividad e inactividad deben cumplir con la siguiente propiedad de cerradura:

$$
{ }_{n}^{a}{ }_{x}^{a}+{ }_{n} L_{x}^{i}={ }_{n} L_{x}
$$

Los años netos de vida activa cumplen con el principio de cerradura por el que las salidas de la cohorte sólo ocurren por mortalidad, es decir, la generación de la tabla no experimenta migraciones a lo largo de su existencia (Partida-Bush, 2000).

\section{TABLA DE VIDA ACTIVA:}

La tabla de vida activa es una combinación de la tabla de vida (biológica) y de las condiciones de actividad prevalecientes en una población en una época dada. Reproduce las condiciones teóricas a que estaría sometida una generación si el nivel de mortalidad y la participación en la vida activa no cambiaran en el futuro (Elizaga, 1979; Partida-Bush, 1996; 2014).

La tabla de vida activa de estados múltiples, como señala PartidaBush (2000) “...es un modelo probabilístico que describe la historia de la presencia en dos estados de una cohorte, generalmente ficticia hasta la muerte del último sobreviviente, bajo los siguientes cuatro supuestos. Markoviano: Las propensiones a moverse entre los estados sólo dependen del estado de presencia al inicio de un intervalo de edades y no de las situaciones previas de la persona. Homogeneidad: Esas propensiones son iguales para todos los sobrevivientes en el mismo estado al inicio de un intervalo de edades. Independencia estocástica: La propensión a moverse entre dos estados no depende 
de otro tipo de movimientos dentro del mismo intervalo de tiempo. Cerradura: Las salidas de la cohorte sólo ocurren por mortalidad, es decir, la generación de la tabla no experimenta migraciones a lo largo de su existencia. Estos cuatro supuestos se adoptan, por un lado, debido a la falta de datos que permitan evitarlos y, por otro, porque simplifican los procedimientos para construir la tabla de vida activa" (p.3).

Para su elaboración utilizamos al igual que en los años netos de vida activa, las tasas de actividad por edad $A_{n}$.

\section{LAS FUNCIONES DE LA TABLA DE VIDA ACTIVA:}

- Sobrevivientes activos e inactivos a la edad exacta $\mathrm{x}$ :

Los sobrevivientes de la tabla se pueden dividir entre activos e inactivos:

$$
l_{x}=l_{x}^{a}+l_{x}^{i}
$$

Por ejemplo, calculamos los sobrevivientes activos de la siguiente forma:

$$
l_{x}^{a}=l_{x}^{*} a_{x}
$$

Donde $\alpha_{x}$ corresponde a la tasa corregida o instantánea de actividad, que se calcula de la siguiente manera: ${ }^{19}$

para intervalos etarios adyacentes de longitud distinta:

$\alpha_{x}=\frac{n{ }_{h} A_{x-h}+h A_{n}}{h+n}$

Y para la misma longitud:

$\alpha_{x}=\frac{A_{x-h}+{ }_{n} A_{x}}{2}$

El resto de funciones se articulan según el siguiente supuesto fundamental:

1) $\mathrm{Si} \mathrm{a}_{\mathrm{x}+\mathrm{n}}>\alpha_{x}$ Solo hay ingresos a la actividad

La ausencia de retiros de la actividad $\left({ }_{n} \mathrm{H}_{\mathrm{x}}^{a i}=0\right)$, las probabilidades de transición al estado puro son:

$$
\begin{array}{ll}
{ }_{n} p_{x}^{a a}=1 & { }_{n} p_{x}^{a i}=0 \\
{ }_{n} p_{x}^{i a}=\frac{\alpha_{x+n}-\alpha_{x}}{i x} & { }_{n} p_{x}^{i i}=\frac{i_{x+n}}{i_{x}}
\end{array}
$$


- Los años-persona vividos:

$$
\begin{array}{ll}
{ }_{n} L_{x}^{a a}={ }_{n} L_{x} \alpha_{x} & { }_{n} L_{x}^{i a}={ }_{n} L_{x}^{a}-{ }_{n} L_{x} \alpha_{x}={ }_{n} L_{x}\left({ }_{n} a_{x}-\alpha_{x}\right) \\
{ }_{n} L_{x}^{a i}=0 & { }_{n} L_{x}^{i i}={ }_{n} L_{x}^{i}-{ }_{n} L_{x}^{a i}={ }_{n} L_{x}^{i}={ }_{n} L_{x}{ }_{n}{ }^{i}
\end{array}
$$

Donde ${ }_{n} L_{x}^{a a}$ son los ańos-persona vividos en la actividad por los activos de edad $\mathrm{x}, \mathrm{y}_{n} a_{x}$ es la proporción de participación de activos para el grupo de edades en la tabla de vida activa. Siendo ${ }_{n} a_{x}={ }_{n} A_{x}$ si $A_{n}>\alpha_{x} / / \frac{\alpha_{x}+\alpha_{x n}}{2}$ si $A_{n}<\alpha_{x}$

- Ingresos:

$$
{ }_{n} H_{x}^{i a}=l_{x+n}^{a}+l_{x}^{a}+{ }_{n} d_{x}^{a} \mathrm{o}{ }_{n} H_{x}^{i a}=l_{x}^{i}+l_{x+n}^{i}+{ }_{n} d_{x}^{i}
$$

2) Si $a_{x+n}<\alpha_{x}$ Solo hay retiros de la actividad

Dada la condición no hay ingresos a la actividad $\left({ }_{n} H_{x}^{i a}=0\right)$, las probabilidades de transición al estado puro son:

$$
\begin{array}{ll}
{ }_{n} p_{x}^{a a}=\frac{\alpha_{x+n}}{\alpha_{x}} & { }_{n} p_{x}^{a i}=\frac{\alpha_{x} \alpha_{x+n}}{\alpha_{x}} \\
{ }_{\mathrm{n}} \mathrm{p}_{x}^{\mathrm{ia}}=0 & { }_{\mathrm{n}} \mathrm{p}_{x}^{\mathrm{ii}}=1
\end{array}
$$

- Los años-persona vividos:

$$
{ }_{n} L_{x}^{a a}={ }_{n} L_{x}^{a}-{ }_{n} L_{x}^{i a}={ }_{n} L_{x}^{a}={ }_{n} L_{x} a_{x} \quad{ }_{n} L_{x}^{i a}=0
$$

Siendo ${ }_{n} a_{x}={ }_{n} A_{x} \sin n A x<\alpha_{x} / / \frac{a_{x+2} \alpha_{x+n}}{2}$ si $A_{n}>\alpha_{x}$

- retiros:

${ }_{n} H_{x}^{a i}=l_{x}^{a}-l_{x+n}^{a}-{ }_{n} d_{x}^{a} \mathrm{O}{ }_{n} H_{x}^{a i}=l_{x+n}^{i}-l_{x}^{i}+{ }_{n} d_{x}^{i}$

Esperanza de vida

$$
e_{x}^{a a}={ }_{n} e_{x}^{a a}+{ }_{n} p_{x}^{a a} e_{x+n}^{a a}+{ }_{n} p_{x}^{a i} e_{x+n}^{i a}
$$

Donde $e_{x}^{a a}$ corresponde a los años-persona vividos per cápita por situación de cada intervalo etario. Debido a que en nuestra tabla no contemplamos activos a partir de los 80 años, $e_{80}^{a a}=0$. 


\section{- Tasas de ingreso, retiro y muerte en orden respectivo: ${ }_{n} m_{x}^{i a}=\frac{{ }_{n}^{H_{x}^{i a}}}{{ }_{n} L_{x}^{i}} ;{ }_{n} m_{x}^{i a}=\frac{{ }_{n} H_{x}^{a i}}{{ }_{n} L_{x}^{a}} ;{ }_{n} m_{x}=\frac{{ }_{n} d_{x}}{{ }_{n} L_{x}}$}

\section{Notas}

${ }^{1}$ El presente artículo corresponde a una edición de capítulo 6 de mi tesis de doctorado titulada: "El cambio demográfico en Chile y sus efectos sobre la fuerza de trabajo (1934-2006)", realizada en el marco del programa de Doctorado en Historia Económica de la Universidad de Barcelona, 2016.

${ }^{2}$ En nuestro caso, pese a que la edad legal a partir de la cual los sistemas de seguridad social en Chile permiten acceder a prestaciones por vejez entre las que se encuentra la jubilación, hemos establecido como punto d al grupo de 80 y más ańos, con la finalidad de conocer las condiciones de retiro tras la edad límite legal.

${ }^{3}$ Estas tablas fueron elaboradas sobre la base de una revisión crítica de los datos disponibles para su elaboración, en cuanto a la mortalidad, los registros de nacimientos y poblaciones censadas, además de una revisión bibliográfica exhaustiva de la bibliografía sobre el tema. Por último, este documento cuenta con una explicación detallada tanto de los métodos utilizados en su elaboración así como de las decisiones tomadas. Ver INE, (2004).

${ }^{4} \mathrm{La}$ imposibilidad de contar con tablas de mortalidad con respaldo institucional para las comunas que componen el Gran Santiago nos obliga a asumir que la mortalidad de este conglomerado es igual a la de la Región Metropolitana -en adelante RM- en su conjunto. Si bien existen departamentos de la RM que no se comprenden en el Gran Santiago, nuestro supuesto radica en el hecho que la mayor parte de la PEA reside dentro de las comunas del Gran Santiago, determinado así el comportamiento de las tablas de mortalidad para la RM. Según el censo de 1982 el 92,6\% de la población entre los 15 y 64 de la RM residía dentro de las comunas que componen el Gran Santiago.

${ }^{5}$ Ver, Hurtado Ruiz-Tagle (1966); De Ramón (1992).

${ }^{6}$ En el censo de 2002 la población en edad de trabajar que reside en medios urbanos alcanza un $85,5 \%$ del total de población entre los 15 y 64 ańos.

7 Ver Fucaraccio (1974); Pardo, (1988); Paredes (2003); Larrańaga (2006); Cerda (2007).

8 Ver Arellano (1981).

9 Si en 1960, en la Región Metropolitana, la esperanza de vida restante para el grupo 60-64 se calculaba en 16 ańos, en 2002 esta había aumentado en 6 ańos, alcanzando una esperanza de vida de 22 ańos. Ver INE (2004).

${ }^{10}$ Ciertamente antes de los 30 ańos, se pueden producir retiros pero su efecto es anulado por los ingresos al mercado de trabajo.

${ }^{11}$ La edad mínima legal para entrar al mercado de trabajo en Chile se definió sobre la base de los años de obligatoriedad de educación. En este sentido, desde 1957 esta se extiende de 6 a 10 ańos, abarcando hasta los 13 ańos de edad. De la misma manera la EOD recoge a la población activa e inactiva a partir de los 14 y más años.

${ }^{12}$ Con una perspectiva de futuro, el aumento de los ańos de vida en inactividad, gracias al aumento de la esperanza de vida a la edad de retiro del mercado laboral, hace necesario un contar con un sistema de seguridad social adecuado, que sea capaz de dar respuesta a las necesidades de las personas en su retiro (Riesco, 2006; 2009).

${ }^{13}$ Por ejemplo, Wolfbein (1949), en su estudio sobre la duración de la vida activa en estados Unidos, estimó para 1940 una EVA de los trabajadores hombres a los 15 años de 45,7 años. Por su parte Chile en el periodo 2001-02 muestra una EVA masculina de 41,6 ańos a los 15 ańos. 
Cabe recordar que en el cálculo de la EVA se reflejan tanto las condiciones del mercado de trabajo como las condiciones de mortalidad a las que se ve sometida la fuerza de trabajo en el momento del análisis. De esta manera los resultados de la EVA en Chile están en línea como los observados en otros países latinoamericanos como Argentina, México y Costa Rica (ver Partida-Bush, 1996; 2000).

${ }^{14}$ Entiéndase como "indicador biosocial de bienestar", la esperanza de vida al nacer o a una edad determinada. Asimismo las tasas de mortalidad general e infantil y los niveles de fecundidad de la población, pero también otros menos frecuentes como la estatura media de la población. Todos estos indicadores pueden ser interpretados como signos de progreso socioeconómico especialmente útiles cuando se utilizan en perspectiva histórica. Ver Cámara, (2014).

${ }^{15}$ Ver Sadie, (1964).

${ }^{16}$ Los ańos brutos como netos de vida activa muestran fluctuaciones en las tendencias a través de los periodos que son reflejo de las tasas de participación de periodo observadas en la EOD, ver Cuadro 3 en apéndice.

${ }^{17}$ Como hemos señalado anteriormente respecto de la elaboración de las tablas de vida, la EVA supone que las condiciones de teóricas a las que estaría sometida una generaciones si el nivel de mortalidad y de participación se mantuvieran constante. Ver Elizaga (1970; 1979), PartidaBush (2000; 2014).

${ }^{18}$ Ver Chackiel, (2000).

${ }^{19}$ La tasa instantánea se refiere a un punto del intervalo, generalmente el punto inicial del intervalo (por ejemplo, 10, 15, 20 etc.). Debido a que las tasas promedio de actividad ${ }_{n} A_{x}$ no siempre son del todo regulares se procese a corregir a través de la interpolación de la que resulta. La actividad económica empieza en el primer intervalo de edad considerado, siendo la tasa igual a 0 en el momento inicial o punto de partida (Elizaga, 1979).

\section{Fecha de Recepción del Artículo: 16 dee septiembre de 2017 FeCha DE ACEPTACIÓn: 30 de noviembre de 2017}

